\title{
A SURVEY OF REPRODUCTIVE BIOLOGY IN NEOTROPICAL MELASTOMATACEAE AND MEMECYLACEAE'
}

\author{
Susanne S. Renner ${ }^{2}$
}

\section{ABSTRACT}

In the Neotropics, the Melastomataceae and Memecylaceae comprise over 3,000 species in 106 genera. Pollination observations have been reported for 126 species in 35 genera of Melastomataceae and for four species in one genus of Memecylaceae. Genetic self-incompatibility has been found in 22 Melastomataceae species, self-compatibility in 25 species. A single Memecylaceae species tested is self-compatible. Agamospermy is known in 19 New World and some Old World species of Melastomataceae. The incidence of polyploidy in Melastomataceae seems high, but only about $8 \%$ of the species have been counted. Most Melastomataceae and Memecylaceae are hermaphroditic; dioecism is known in a few species in one genus of Melastomataceae. The principal mode of promoting outcrossing in both families is spatial separation of pollen and stigma (herkogamy), achieved by the pollen being enclosed in poricidal anthers, which have to be manipulated to release pollen. New World Memecylaceae are pollinated in the same way and by some of the same bee species as most Melastomataceae. All bee species known to visit Melastomataceae and Memecylaceae are listed; they comprise a wide spectrum of the neotropical bee fauna. It is not yet clear whether the stamen dimorphism and the conspicuous connective appendages present in many Melastomataceae have a function in the pollination mechanism beyond that of enhancing the visual attractiveness of the flowers and making the stamens easier to grasp. In most Melastomataceae, flowers offer only pollen; however, some 60 species in 8 genera offer nectar as a reward for pollinators (probably, some species in at least one additional genus do, too). The nectariferous species are pollinated by birds, bats, rodents, and bees. The nectar is rich in sucrose and, as a rule, its production is correlated with floral morphological changes relating to the fact that, except for bees, nectar consumers do not vibrate the stamens to collect pollen actively. I suggest that the capacity for developing nectaries is basic in Melastomataceae but suppressed in most modern members. The staminal glands of Memecylaceae produce minute quantities of a secretion containing lipids which, however, is not collected by the pollinators; the glands may in some way enhance stamen attractiveness. In seed dispersal systems, major diversifications occurred, with $40 \%$ of the neotropical Melastomataceae having capsular fruits and wind-dispersed seeds and $60 \%$ having soft, juicy berries taken mainly by birds, but also by marsupials, monkeys, bats, other mammals, turtles, and other reptiles. All Memecylaceae have berries, and their seeds are dispersed by birds, monkeys, and fish. I conclude that the east and west Gondwanian lineages of Melastomataceae and Memecylaceae, in parallel, coevolved with the earliest bees in the lower mid. Cretaceous. This may have been the time when the ancestors of these families switched from nectar-reward flowers to pollen-reward flowers. In the further extensive radiation of both families, special adaptations to pollinators were of limited importance; diversification in seed dispersal systems, vegetative characters, and edaphic adaptations were more important.

The neotropical Melastomataceae and Memecylaceae are particularly suitable for a study of reproductive biology in view of the relative ease with which members of these families are found in the field and the availability of several recent treatments in monographs and floras. As Gleason (1932) stated, "a dozen or more species . . . grow naturally on every square mile of tropical America, unless

' I thank the following apidologists for identifying bees: M. C. Almeida (Trigona), R. Dressler (Euglossini), G. Eickwort (Halictidae), V. Graaf (Oxaea), C. D. Michener (Exomalopsini), S. Laroca and J. R. Cure Hakin (Halictidae), J. Moure (Xylocopini, Monoeca, Meliponini), D. Roubik (Melipona, Trigona), R. Snelling (Centridini, Ceratini), and D. Urban (Eucerini). Except for specimens retained by the specialists, bees are deposited in the entomological collection of the Instituto Nacional de Pesquisas da Amazonia (INPA). It is a pleasure to acknowledge the support I received at INPA, especially from Drs. W. Rodrigues, M. F. da Silva, and M. L. Absy, also from R. Bierregård and J. Rankinde-Merona, zoological and botanical field directors, respectively, of the INPA.WWF "Minimal Critical Size of Ecosystems" project. Fieldwork in Brazil was supported by the Deutscher Akademischer Austauschdienst and the Deutsche Forschungsgemeinschaft. Fieldwork in Venezuela was funded by grants from the National Science Foundation and the Smithsonian Scholarly Studies Program. I am grateful to Klaus Kubitzki and John Wurdack for their help during different phases of this work and to T. Morley, C. Todzia, D. Roubik, H. Tobe, B. Stein, R. Lundin, and K. Bremer for comments on the manuscript.

Botanical Institute, University of Aarhus, Nordlandsvej 68, DK-8240 Risskov, Denmark.

Ann. Missouri Bot. Gard. 76: 496-518. 1989. 
Table 1. New World Melastomataceae and Memecylaceae. Tribal arrangement following Cogniaux (1891), except for more recently described, transferred, or synonymized genera. Species numbers are those of the most recent treatment, or based on a herbarium survey (Renner, 1986). + indicates a recorded observation, citation in Table 4; - no observation presently known.

\begin{tabular}{cc}
\hline \hline & $\begin{array}{c}\text { Polli- } \\
\text { nation }\end{array}$ \\
Species & Obser- \\
Number & vation \\
\hline
\end{tabular}

\begin{tabular}{|c|c|c|}
\hline \multicolumn{3}{|l|}{ Melastomataceae } \\
\hline \multicolumn{3}{|l|}{ BERTOLONIEAE } \\
\hline Bertolonia & 8 & - \\
\hline Boyania & 1 & - \\
\hline Diplarpea & l & - \\
\hline Macrocentrum & 21 & - \\
\hline Maguireanthus & 1 & - \\
\hline Monolena & 15 & - \\
\hline Opisthocentra & l & + \\
\hline Salpinga & 8 & + \\
\hline Tateanthus & 1 & - \\
\hline Triolena (incl. Diolena) & 22 & - \\
\hline Tryssophyton & 1 & - \\
\hline \multicolumn{3}{|l|}{ MERIANIEAE } \\
\hline Acanthella & 2 & - \\
\hline Adelobotrys & 25 & + \\
\hline Axinaea & 30 & - \\
\hline Behuria & 3 & - \\
\hline Benevidesia & 1 & - \\
\hline Bisglaziova & 1 & - \\
\hline Centronia & 15 & + \\
\hline Dolichoura & 1 & - \\
\hline $\begin{array}{l}\text { Graffenrieda (incl. Calyptrella } \\
\text { and Ptilanthus) }\end{array}$ & 44 & + \\
\hline Huberia & 6 & - \\
\hline Meriania & 74 & + \\
\hline Merianthera & 3 & - \\
\hline Neblinanthera & 1 & - \\
\hline Ochthephilus & 1 & - \\
\hline Phainantha & 4 & - \\
\hline Tessmannianthus & 3 & - \\
\hline \multicolumn{3}{|l|}{ MICROLICIEAE } \\
\hline Bucquetia & 3 & - \\
\hline Cambessedesia (incl. Pyramia) & 21 & - \\
\hline Castratella & 1 & - \\
\hline Centradenia & 6 & + \\
\hline Centradeniastrum & 1 & - \\
\hline Chaetostoma & 12 & - \\
\hline Eriocnema & 1 & - \\
\hline Lavoisiera & 46 & + \\
\hline Lithobium & 1 & - \\
\hline Microlicia & 100 & - \\
\hline Poteranthera & 2 & - \\
\hline Rhynchanthera & 15 & + \\
\hline Siphanthera (incl. Farringtonia) & 16 & + \\
\hline Stenodon & 1 & - \\
\hline Trembleya & 11 & + \\
\hline \multicolumn{3}{|l|}{ TIBOUCHINEAE } \\
\hline Aciotis & 30 & + \\
\hline Acisanthera & 17 & + \\
\hline Appendicularia & 1 & - \\
\hline Brachyotum & 50 & + \\
\hline
\end{tabular}

TABLE 1. Continued.

\begin{tabular}{|c|c|c|}
\hline & $\begin{array}{l}\text { Species } \\
\text { Number }\end{array}$ & $\begin{array}{l}\text { Polli- } \\
\text { nation } \\
\text { Obser- } \\
\text { vation }\end{array}$ \\
\hline Chaetolepis & 10 & - \\
\hline Comolia & 22 & - \\
\hline Comoliopsis & 1 & - \\
\hline Desmoscelis & l & + \\
\hline Ernestia & 16 & + \\
\hline Fritzschia & 1 & - \\
\hline Heterocentron & 6 & - \\
\hline Loricalepis & l & - \\
\hline Macairea & 22 & + \\
\hline Mallophyton & 1 & - \\
\hline Marcetia & 23 & - \\
\hline Microlepis & 4 & - \\
\hline Nepsera & l & + \\
\hline Pilocosta & 3 & - \\
\hline Pseudernestia & 1 & - \\
\hline Pterogastra & 4 & + \\
\hline Pterolepis & 15 & - \\
\hline Sandemania & 1 & + \\
\hline Schwackaea & l & - \\
\hline Svitramia & l & - \\
\hline $\begin{array}{l}\text { Tibouchina (incl. Itatiaia and Pur- } \\
\text { purella) }\end{array}$ & 243 & + \\
\hline Tibouchinopsis & 2 & - \\
\hline \multicolumn{3}{|l|}{ RHEXIEAE } \\
\hline Monochaetum & 45 & + \\
\hline Pachyloma & 6 & - \\
\hline Rhexia & 13 & + \\
\hline \multicolumn{3}{|l|}{ MICONIEAE } \\
\hline Anaectocalyx & 3 & - \\
\hline Bellucia & 7 & + \\
\hline Calycogonium & 23 & - \\
\hline Catacoryne & 1 & - \\
\hline Chalybea & 1 & + \\
\hline Charianthus & 11 & - \\
\hline Clidemia & 117 & + \\
\hline Conostegia & 43 & - \\
\hline Ekmaniocharis & 1 & - \\
\hline Henriettea & 12 & + \\
\hline Henriettella & 51 & + \\
\hline Heterotrichum & 10 & - \\
\hline Huilaea & 4 & + \\
\hline Killipia & 4 & - \\
\hline Kirkbridea & l & - \\
\hline Leandra (incl. Platycentrum) & 175 & + \\
\hline Llewellynia & 1 & - \\
\hline Loreya & 13 & + \\
\hline Maieta & 2 & - \\
\hline Mecranium & 21 & - \\
\hline ca. 1,000 (at least & st 958$)$ & + \\
\hline Mommsenia & 1 & - \\
\hline Myriaspora & 1 & + \\
\hline Myrmidone & 2 & - \\
\hline Necramium & 1 & - \\
\hline Ossaea & 91 & - \\
\hline Pachyanthus & 16 & - \\
\hline Pleiochiton & 7 & - \\
\hline Tetrazygia & 21 & - \\
\hline Tococa & 54 & + \\
\hline
\end{tabular}


TABLE 1. Continued.

\begin{tabular}{lrc}
\hline \hline & & $\begin{array}{c}\text { Polli- } \\
\text { nation } \\
\text { Species } \\
\text { Obser- } \\
\text { Number }\end{array}$ \\
vation \\
\hline CYPHOSTYLEAE & 1 & - \\
Allomaieta & 7 & - \\
Alloneuron & 1 & - \\
Cyphostyla & 100 & + \\
BLAKEEAE & 62 & + \\
Blakea & & \\
Topobea & 81 & + \\
MEMECYLACEAE & 8 & - \\
Mouriri & & \\
Votomita & & \\
\hline
\end{tabular}

the land is under intense cultivation, and the various genera extend from the coastal marshes at sea-level to the high páramos above the tree line." The Melastomataceae are a large family (v.i.) and diverse in terms of habit, reproductive and vegetative architecture (Cremers, 1983), and indument (Wurdack, 1986). Memecylaceae comprise about 89 species in the Neotropics and are much less diverse in these characters.

The present survey is based on my observations of the reproductive biology of many Brazilian and some Venezuelan species and on a review of the literature. The survey is presented in five sections. First, an outline of the classification and reproductive morphological features of the Melastomataceae and Memecylaceae is given; second, breeding systems and cytology are discussed; third, the pollination spectra found in the two families are described; fourth, a brief survey is made of seed dispersal in the two families; fifth, the reproductive biological information is related to the fossil record and distributional data to contribute to a better understanding of the historical evolution of the two families.

Flowering and fruiting phenology of neotropical melastomes and Memecylaceae are not dealt with here, but reference may be made to Snow (1965), Croat (1978), Opler et al. (1980), Hilty (1980), Lumer (1982), and Renner (1984 and in prep.). Suffice it to say that synchronous, episodic flowering with the episodes of short duration (a few days) characterizes some of the arborescent species of the largest genus, Miconia, whereas extended flowering periods (several weeks or months) are characteristic of many of the shrubs, herbs, epiphytes, and some of the tree species.

\section{Classification and Reproductive}

\section{Morphological Features}

Worldwide, the Melastomataceae A. L. Juss. (excluding the ca. ten species of Crypteronia, Axinandra, and Dactylocladus, i.e., the Crypteroniaceae; Dahlgren \& Thorne, 1984) and the Memecylaceae DC. consist of ca. 190 genera and approximately 4,800 species. In the Neotropics, the Melastomataceae comprise over 3,000 species in 107 genera (Table 1) and the Memecylaceae 89 species in two genera. About half of these genera contain only one to four species, whereas one genus, Miconia, has some 1,000 species. Though the Memecylaceae are often included in the Melastomataceae as a subfamily, it has been pointed out (see Morley, 1953; Dahlgren \& Thorne, 1984; Johnson \& Briggs, 1984) that the differences between these families are such that their union obscures rather than reflects our knowledge of the two groups. The Memecylaceae consist of the Asian genera Memecylon (ca. 300 spp.) and Lijndenia (4 spp.) (Bremer, 1982, 1983), the African Spathandra (6 spp.) and Warneckea (3l spp.), and the neotropical Votomita (8 spp.; Morley, 1963) and Mouriri (81 spp.). There is a consensus that the Melastomataceae and Memecylaceae belong to the core families of the order Myrtales (Dahlgren \& Thorne, 1984; Johnson \& Briggs, 1984).

The classification of the Melastomataceae sensu stricto is essentially that of Triana (1867), who was closely followed by Cogniaux (1891). W urdack (1980) has realigned the New World tribes in a sequence generally resembling that of $\mathrm{A}$. P. de Candolle (1828). The main dichotomy in the Melastomataceae is between the capsular-fruited and the berry-fruited genera. The genera are arranged into tribes on the basis of fruit and seed characters and the presence and position of connective appendages (D. Don, 1823; Triana, 1867; Cogniaux, 1891; Gleason, 1929; comp. also Whiffin \& Tomb, 1972).

Most Melastomataceae are characterized by poricidal anthers, the connectives of which are frequently prolonged (however, species of Miconia sects. Chaenopleura and Chaenanthera have anthers that open by $1-2$ slits). They usually have a pronounced foliar venation consisting of the midvein and one to four pairs of subparallel longitudinal veins. Memecylaceae, on the other hand, mostly have pinnate venation; their anthers open by short slits or pores. In a reproductive biological context, the presence of an elliptic, concave gland on the dorsal side of the connective is a unique feature characterizing most Memecylaceae. 
The flowers in both families are actinomorphic or weakly to strongly zygomorphic as a result of movements of the stamens and style immediately after the expansion of the petals (Troll, 1922; Ziegler, 1925; pers. obs.). In Melastomataceae, the flowers are usually borne in well-developed cymose inflorescences, whereas in Memecylaceae the cymes are often condensed or reduced to a few flowers in fasciculate tufts. In both families, the mostly 4 - or 5 -merous flowers are perigynous or epigynous with a cup-shaped hypanthium that bears on its rim the calyx lobes, petals, and stamens. The antepetalous stamens are usually longer than the antesepalous ones, which may be staminodial; this dimorphism is more or less pronounced and has given occasion to much speculation concerning its role in pollination. In Melastomataceae with poricidal anthers, the anthers are one- or twopored, a character that is consistent at the species level but varies within genera; there is also considerable variation in the size and position of the pores. Pollen grains are usually free, small, and rather smooth, although polyads and tetrads are known in species of Tococa and Miconia (Patel et al., 1984).

\section{BREEding Systems}

The principal mode of promoting outcrossing in most Melastomataceae and Memecylaceae is the very effective spatial separation of pollen and stigma in the flowers (herkogamy). This is achieved by the pollen being enclosed in tubular anthers with apical or subapical pores or slits, which are generally minute, and positioned well distant from the punctiform or capitate stigmas. Also, the stamens are usually shorter than the styles. In bud, the stamens are always folded over in such a way that the anther pores point downward and are maximally distant from the stigma, which lies at the tip of the bud. Thus, chances for automatic selfing before and during anthesis are very low or nil. If, however, during anthesis, an anther or the style bends in such a way that the pore is brought into contact with the stigma, automatic selfing may occur. This has been reported for Monolena trichopoda (Melastomataceae; Warner, 1981) where, late in anthesis, the stamens fold over and the thecae are effectively stuck to the stigmatic exudate.

No pollen escapes from the anthers unless mediated by a vector, which at least potentially may arrive bearing conspecific pollen. Almeda (1977, 1978) suggested that vigorous mechanical move- ment of the anthers by gusty winds or rains might release pollen in species of Monochaetum and Centradenia (Melastomataceae). Additionally, he often observed pollen in water droplets connecting anthers and stigma and suggested that this might be another possible route for selfing to occur. However, bees, which regularly collect pollen from Melastomataceae, use vibrations of around $420 \mathrm{~Hz}$ or higher (this frequency was found to effect ample pollen emission from the large anthers of Bellucia; see below). Therefore, it seems unlikely that the low-frequency vibrations produced by wind could release pollen from melastome anthers. This impression is supported by the lack of fruit set in bagged flowers of nonagamospermic species exposed to strong wind (Renner, 1984). While autogamy may be extremely rare, vector-mediated selfing (i.e., geitonogamy), a function of pollinator behavior and number of conspecific flowers available at a time, may be frequent in self-compatible species.

Temporal separation of male and female function has been suggested for species of Monochaetum (Almeda, 1978) in which "the ill-defined stigmatic region ... appears to be non-receptive during the first day." This may be a rare condition because, as a rule, poricidal flowers that offer only pollen as a reward (subsequently also called "pollen-only flowers") receive foreign pollen at the same time a visitor harvests pollen from them; in femalephase flowers, there is nothing to reward visitors (only mimicry or deception might account for visits to female-phase flowers). Homogamy, i.e., the simultaneous functioning of the male and female parts, is thus to be expected in pollen-only flowers. Temporal separation of the male and female function in single flowers has been reported twice in nectariferous melastomes (Ule, 1896; Vogel, 1957); in both cases, the flowers were protandrous.

The Melastomataceae are not entirely hermaphroditic. Wurdack (1964) was the first to describe dioecism in Miconia dioica Wurdack; herbarium specimens of this species had female flowers with abortive stamens and male flowers with poorly developed stigmas. $\mathrm{He}$ also suspected dioecism in some other minute-flowered Andean species of $M i$ conia (M. dielsii Markgraf, M. clathrantha $\mathrm{Tr}$. ex Cogn., M. minuta Gleason; Wurdack, 1980: 261-262) and in M. rubens (Sw.) Naudin from the West Indies and Venezuela (Wurdack, 1964). Field observations on the reproductive biology of these and some other suspected dioecious melastomes are lacking.

At least one species of Lijndenia (Memecyla- 
ceae) has hermaphrodite-flowered individuals and purely male-flowered ones (Bremen, 1982).

\section{GENETIC COMPATIBILITY}

As shown in Table 2, genetic self-incompatibility occurs in at least 22 species in the two families. As Darwin remarked (in a letter to J. D. Hooker on Nov. 28, 1871, in F. Darwin (ed.), 1903), melastomes "are troublesome beasts to fertilise." This is due to the closed anthers and mostly punctiform stigmas. In some large-flowered species, such as Bellucia (Melastomataceae), it is possible to collect large amounts of pollen for experimental purposes by holding a tuning fork to the stamens. Self-compatibility is found in 26 species (Table 2). Since no efforts have ever been made to distinguish pseudogamy from self-compatibility, several of the supposedly self-compatible species reported earlier (Darwin, 1876; Ziegler, 1925) may have set fruit as a result of agamospermy rather than of selffertilization. Related species such as Tococa guianensis and T. bullifera or Centradenia grandiflora and C. paradoxa (all Melastomataceae) differ in their compatibility conditions (Table 2). The humid stigmatic surface and the binucleate pollen of the Melastomataceae and Memecylaceae (Tobe \& Raven, 1984) indicate that the incompatibility system is gametophytic (Nettancourt, 1977).

\section{AGAMOSPERMY}

Experimental proof of agamospermy in melastomes is readily obtained by cutting off the style in freshly opened unvisited flowers. (To ensure that flowers are unvisited before the style is cut off, they must be bagged the evening before.) Apart from the 22 apomictic Melastomataceae listed in Table 3, polyembryony, with additional embryos being formed by nucellus or suspensor cells, has been reported from the Asiatic species Osbeckia hispidissima Wight, Sonerila wallichii Benn., and Melastoma malabathricum L. (Subramanyam, 1942, 1944, 1948). Polyembryony is often correlated with agamospermy (Gustafsson, 1946-47). Mouriri nervosa is the only member of the Memecylaceae so far tested for agamospermy, with negative results (Renner, unpubl.). In several of the neotropical species showing apomixis, for instance in Clidemia novemnervia and Macairea theresiae, the anthers contain very few pollen grains. In cultivated neotropical Leandra cordifolia Cogn., Subramanyam (1942) also found that a low percentage of fertile pollen grains was combined with the frequent formation of two embryo sacs. In most neotropical species, agamospermy is correlated with low stainability of fresh pollen in cotton blue with lactophenol. This stain indicates presence of callose, a saccharide found in the intine of pollen. Lack of an intine could make a grain unviable and is usually accompanied by lack of cytoplasm. However, in Aciotis acuminifolia and Miconia argyrophylla, pollen grains stain heavily, yet these species are capable of producing seeds apomictically. In Clidemia novemnervia and Maieta poeppigii, the stamens do not unflex completely upon anthesis but remain curved, and are thus not available for legitimate bee pollinators. Both species regularly set seeds apomictically.

Lumer (1982) observed that Blakea gracilis, Blakea sp. (originally identified as B. grandiflora), and Topobea brenesii ripened a large number of seedless fruits from emasculated flowers; however, in T. pittieri, she found that of the bagged flowers, $35 \%$ produced mature fruits containing seeds. Darwin (1876) and Ziegler (1925) observed fruit set in cultivated plants of Monochaetum, Tibouchina, Centradenia, Calvoa, and Dissotis in the greenhouse. Assuming that there were no bee pollinators in the greenhouses, it is likely that such fruits resulted from agamospermy rather than from autogamy, which, as pointed out above, is mechanically difficult in Melastomataceae.

The number of species investigated is still too small to draw firm conclusions about correlations between habit, habitat, and breeding system. Of the agamospermous species, 13 are shrubs of various types of savannas or of secondary vegetation; five are treelets, two are herbs, and one is a climber. The sample is clearly biased towards shrubby species, however, because of their accessibility.

\section{Cytology}

Some 300 of the over 3,000 species of neotropical Melastomataceae and only one species of Memecylaceae (Mouriri myrtilloides) are known cytologically (Solt \& Wurdack, 1980). Only a few of these have been examined twice. Melastome chromosomes are very small (0.5-1 micron; Solt \& Wurdack, 1980) and stain poorly. The most common base numbers are 9 and 17 (Wurdack \& Kral, 1982). Based on Solt \& Wurdack's results, the incidence of polyploidy in the Melastomataceae seems high. It cannot yet be answered whether polyploidy is more frequent in species with strong agamospermous reproduction than in those which lack apomixis, nor whether there is a correlation between polyploidy and growth habit.

\section{Pollination Systems}

In neotropical Melastomataceae and Memecylaceae, the most widespread pollination system 
TABLE 2. Compatibility systems in Melastomataceae.

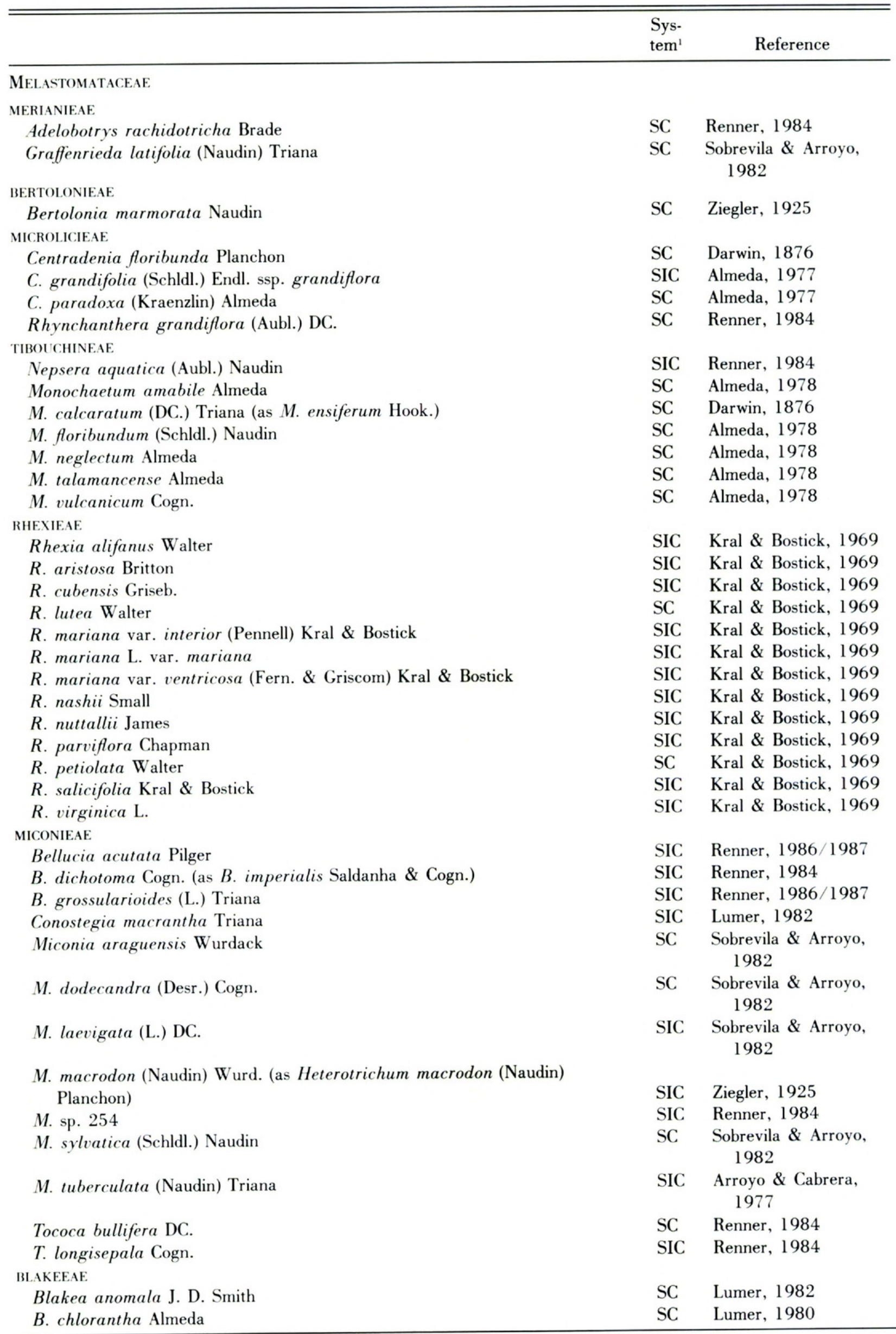


TABle 2. Continued.

\begin{tabular}{|c|c|c|}
\hline & $\begin{array}{l}\text { Sys- } \\
\text { tem }\end{array}$ & Reference \\
\hline B. gracilis Hemsley & $\mathrm{SC}$ & Lumer, 1982 \\
\hline B. sp. (as B. grandiflora Hemsley) & $\mathrm{SC}$ & Lumer, 1982 \\
\hline B. tuberculata J. D. Smith & $\mathrm{SC}$ & Lumer, 1982 \\
\hline Topobea brenesii Standley & $\mathrm{SC}$ & Lumer, 1982 \\
\hline T. durandiana Cogn. & $\mathrm{SC}$ & Lumer, 1982 \\
\hline \multicolumn{3}{|l|}{ MEMECYLACEAE } \\
\hline Mouriri nervosa Pilger & $\mathrm{SC}$ & Renner, 1984 \\
\hline
\end{tabular}

' $\mathrm{SC}=$ self-compatible, $\mathrm{SIC}=$ self-incompatible.

involves female bees; it occurs in an estimated $98 \%$ of the species. Pollination by hummingbirds, bats, and rodents is found in an estimated 60 species of Meriania, Centronia, Brachyotum, Tibouchina, Miconia, Huilaea, Chalybea, and Blakea (Table 4 ), and probably also in Axinaea (see below). The mode of pollination is determined by the type of reward offered: Melastomataceae and Memecylaceae with pollen-only flowers are, as a rule, pollinated exclusively by bees because the morphology of the anthers excludes all other pollen-collecting vectors. In contrast, the nectar-offering species attract a wide variety of pollinators.

\section{MELASTOMATACEAE}

POLLINATION BY POLLEN-COLLECTING BEES

a) Methods of pollen collection and kinds of bees

Pollination by bees has been reported for some 120 species in 31 genera of Melastomataceae (Table 4). In addition, a few species occurring at Finca La Selva, Costa Rica, and near Kourou, French Guiana, have been observed to be pollinated by small and medium-sized bees (Bawa et al. 1985; Roubik, 1979a). Many unidentified melastomes are indicated as visited by bees in apidological studies (e.g., Sakagami et al., 1965, and Roubik, 1979b).

TABle 3. Agamospermous Melastomataceae.

\begin{tabular}{|c|c|c|c|}
\hline & Habit & Habitat & Reference \\
\hline $\begin{array}{l}\text { Aciotis acuminifolia (DC.) } \\
\text { Triana }\end{array}$ & herb & forest understory & Renner, 1984 \\
\hline $\begin{array}{l}\text { Clidemia capitellata (Bonpl.) D. } \\
\text { Don }\end{array}$ & shrub & savannas and disturbed vegetation & Renner, 1984 \\
\hline C. epibaterium DC. & climber & forest understory & Renner, 1984 \\
\hline C. fendleri Cogn. & shrub & forest understory & Sobrevila \& Arroyo, 1982 \\
\hline C. hirta (L.) D. Don & shrub & disturbed vegetation & Renner, 1984 \\
\hline C. novemnervia (DC.) Triana & shrub & disturbed vegetation & Renner, 1984 \\
\hline C. rubra (Aubl.) Mart. & shrub & savannas and disturbed vegetation & Renner, 1984 \\
\hline Macairea theresiae Cogn. & shrub & Amazonian white sand savannas & Renner, 1984 \\
\hline Maieta guianensis (Aubl.) DC & shrub & forest understory & original \\
\hline Maieta poeppigii Cogn. & shrub & forest understory & original \\
\hline Miconia alata (Aubl.) DC. & shrub & disturbed vegetation & Renner, 1984 \\
\hline M. albicans (Sw.) Triana & shrub & savannas (cerrado) & Renner, 1984 \\
\hline M. argyrophylla DC. & treelet & secondary forest & Renner, 1984 \\
\hline M. campestris (Benth.) Tr. & shrub & disturbed vegetation & Renner, 1984 \\
\hline M. egensis Cogn. & treelet & secondary forest & Renner, 1984 \\
\hline M. minutiflora (Bonpl.) DC. & treelet & secondary forest & Renner, 1984 \\
\hline M. prasina $(\mathrm{Sw}.) \mathrm{DC}$ & treelet & secondary forest & original \\
\hline M. regelii Cogn. & treelet & secondary forest & Renner, 1984 \\
\hline M. spinulosa Naudin & shrub & forest understory & Sobrevila \& Arroyo, 1982 \\
\hline M. stenostachya DC. & shrub & savannas & Renner, 1984 \\
\hline M. tomentosa (L. C. Rich.) D. & treelet & disturbed vegetation & Renner, 1984 \\
\hline
\end{tabular}

Don ex DC.

Rhexia mariana L.

herb

swamps, marshes

Etheridge \& Herr, 1968 
TABLE 4. Pollinators of Melastomataceae and Memecylaceae.

\begin{tabular}{lll}
\hline \hline Important Pollinators $^{1}$ & References \\
\hline
\end{tabular}

Melastomataceae

BERTOLONIEAE

Opisthocentra clidemioides

Hook. f.

Salpinga secunda DC.

\section{MERIANIEAE}

Adelobotrys rachidotricha Brade

Centronia phlomoides Triana

Graffenrieda fruticosa Wurdack

G. polymera Gleason subsp. neblinensis Wurd.

G. reticulata Wurdack

Meriania longifolia Cogn.

M. tomentosa (Cogn.) Wurd. (= Centronia excelsa (Bonpl.) DC.)

MICROLICIEAF

Centradenia grandifolia (Schldl.) Endl.

Lavoisiera cordata Cogn.

L. glandulifera Naudin

Rhynchanthera grandiflora (Aubl.) DC.

\section{R. hispida Naudin \\ R. sp.}

Siphanthera cordifolia (Benth.) Gleason

Trembleya phlogiformis DC.

TIBOUCHINEAE

Aciotis acuminifolia (DC.)

Triana

A. annua (DC.) Triana

A. circaeifolia (Bonpl.) Triana

A. polystachya (Bonpl.) Triana

\section{Acisanthera uniflora (Vahl)} Gleason

Brachyotum ledifolium (Desr.) Triana

Brachyotum ca. $40-45$ species

Desmoscelis villosa (Aubl.) Naudin
Euglossa intersecta; Melipona fulva

Euglossa sp., Eulaema nigrita; Meli- $\quad$ Renner, 1984 pona sp.

Pseudaugochloropsis sp.

bats

Bombus spp.; Xylocopa sp.; Melipona lateralis

Bombus spp.; Melipona lateralis

Bombus spp.; Melipona lateralis; Dialictus sp.; Neocorynura sp.

Xylocopa sp.

hummingbirds

Bombus sp.; Melipona sp.

Bombus spp.

Bombus spp.

Xylocopa frontalis, X. tegulata, $X$. muscaria; Centris nitens, C. flavifrons; Eulaema nigrita; Monoeca sp.

Xylocopa transitoria; Centris fuscata; Ptiloglossa sp.

Augochloropsis scabrifrons, A. sparsilis, A. terrestris, Augochloropsis sp.; Pseudaugochloropsis graminea

Dialictus sp.

Thygater analis; Bombus sp.; Xylocopa sp.

Megommation ogilvei; Paratetrapedia duckei; Melipona fulva, Melipona sp.; Exomalopsini halictids; Paratetrapedia sp. Megommation sp.

Paratetrapedia sp.; Augochloropsis callichroa, Augochloropsis sp.; Euglossa sp.

halictids

hummingbirds

hummingbirds

Dialictus sp.; Augochloropsis sp.; Xylocopa cf. tegulata; Augochlora sp.; Melipona sp.
Renner, 1984

Vogel, 1988

Renner, in press

Renner, in press

Renner, in press

Vogel, 1978

van der Werff, pers. comm.; Neill et al., label data

Almeda, 1977

Renner, 1984

original

Renner, 1984, and original

Renner, 1984

Laroca, 1970

N. Ramirez, pers. comm., 1986

Renner, 1984; Semir, pers. comm., 1982

Renner, 1984

Renner, 1984

Renner, 1984

Renner, 1984, and original

original

Lagerheim, 1899

Lagerheim, 1899; Wurdack, 1954; Fitzpatrick et al., 1979; B. Stein, pers. comm., 1985

Roubik, 1979b; Renner, 1984, and original 
Table 4. Continued.

\begin{tabular}{|c|c|c|}
\hline & Important Pollinators ${ }^{1}$ & References \\
\hline Ernestia tenella (Bonpl.) DC. & Augochloropsis sp. & original \\
\hline Macairea pachyphylla Benth. & Melipona sp. & N. Ramirez, pers. comm. 1986 \\
\hline M. parvifolia Benth. & Euglossa sp. & N. Ramirez, pers. comm. 1986 \\
\hline M. rufescens DC. & halictids & original \\
\hline Monochaetum alpestre Naudin & $\begin{array}{l}\text { Bombus sp.; Melipona sp.; Antho- } \\
\text { phoridae }\end{array}$ & Almeda, 1978 \\
\hline M. amabile Almeda & $\begin{array}{l}\text { Bombus sp.; Melipona sp.; Antho- } \\
\text { phoridae }\end{array}$ & Almeda, 1978 \\
\hline M. compactum Almeda & $\begin{array}{l}\text { Bombus sp.; Melipona sp.; Antho- } \\
\text { phoridae }\end{array}$ & Almeda, 1978 \\
\hline M. cordatum Almeda & $\begin{array}{l}\text { Bombus sp.; Melipona sp.; Antho- } \\
\text { phoridae }\end{array}$ & Almeda, 1978 \\
\hline $\begin{array}{l}\text { M. deppeanum (Schldl. \& } \\
\text { Cham.) Naudin }\end{array}$ & $\begin{array}{l}\text { Bombus sp.; Melipona sp.; Antho- } \\
\text { phoridae }\end{array}$ & Almeda, 1978 \\
\hline M. exaltatum Almeda & $\begin{array}{l}\text { Bombus sp.; Melipona sp.; Antho- } \\
\text { phoridae }\end{array}$ & Almeda, 1978 \\
\hline M. floribundum (Schldl.) Naudin & $\begin{array}{l}\text { Bombus sp.; Melipona sp.; Antho- } \\
\text { phoridae }\end{array}$ & Almeda, 1978 \\
\hline M. linearifolium Almeda & $\begin{array}{l}\text { Bombus sp.; Melipona sp.; Antho- } \\
\text { phoridae }\end{array}$ & Almeda, 1978 \\
\hline M. macrantherum Gleason & $\begin{array}{l}\text { Bombus sp.; Melipona sp.; Antho- } \\
\text { phoridae }\end{array}$ & Almeda, 1978 \\
\hline M. neglectum Almeda & $\begin{array}{l}\text { Bombus sp.; Melipona sp.; Antho- } \\
\text { phoridae }\end{array}$ & Almeda, 1978 \\
\hline M. talamancense Almeda & $\begin{array}{l}\text { Bombus sp.; Melipona sp.; Antho- } \\
\text { phoridae }\end{array}$ & Almeda, 1978 \\
\hline M. tenellum Naudin & $\begin{array}{l}\text { Bombus sp.; Melipona sp.; Antho- } \\
\text { phoridae }\end{array}$ & Almeda, 1978 \\
\hline M. trichophyllum Almeda & $\begin{array}{l}\text { Bombus sp.; Melipona sp.; Antho- } \\
\text { phoridae }\end{array}$ & Almeda, 1978 \\
\hline M. vulcanicum Cogn. & $\begin{array}{l}\text { Bombus sp.; Melipona sp.; Antho- } \\
\text { phoridae }\end{array}$ & Almeda, 1978 \\
\hline Nepsera aquatica (Aubl.) Naudin & $\begin{array}{l}\text { Megaloptidia contradicta; Megom- } \\
\text { mation sp.; Melipona sp. }\end{array}$ & Roubik, 1979b, Renner, 1984 \\
\hline $\begin{array}{l}\text { Pterogastra divaricata (Bonpl.) } \\
\quad \text { Naud. }\end{array}$ & halictids & original \\
\hline \multicolumn{3}{|l|}{ Purpurella see Tibouchina } \\
\hline $\begin{array}{l}\text { Sandemania hoehnei (Cogn.) } \\
\quad \text { Wurd. }\end{array}$ & $\begin{array}{l}\text { Megommation sp.; Augochloropsis } \\
\text { callichroa, Augochloropsis sp.; } \\
\text { Melipona fulva }\end{array}$ & Renner, 1984, and original \\
\hline $\begin{array}{l}\text { Tibouchina arborea (Gardner) } \\
\text { Cogn. }\end{array}$ & Bombus morio & F. Mueller, 1873 \\
\hline T. aspera Aubl. & $\begin{array}{l}\text { Xylocopa transitoria; Bombus sp.; } \\
\text { Augochloropsis sp.; Euglossa } \\
\text { sp.; Melipona sp.; Ptiloglossa } \\
\text { sp. }\end{array}$ & Roubik, 1979b, Renner, 1984 \\
\hline T. benthamiana (Gardner) Cogn. & Augochloropsis sp. & original \\
\hline T. clavata (Pers.) Wurd. & $\begin{array}{l}\text { Bombus morio; Pseudaugochlorop- } \\
\text { sis graminea }\end{array}$ & Laroca, 1970 \\
\hline $\begin{array}{l}\text { T. } \text { cleistoflora Ule }(=T \text {. cleisto- } \\
\text { petala Ule, } 1896, \text { nom. } \\
\text { emend. = Itatiaia cleisto- } \\
\text { petala (Ule) Ule, } 1909\end{array}$ & Bombus sp. & Ule, 1896 \\
\hline T. frigidula (DC.) Cogn. & $\begin{array}{l}\text { Bombus sp.; Oxaea flavescens; } \\
\text { Pseudaugochloropsis graminea }\end{array}$ & original \\
\hline $\begin{array}{l}\text { T. gardneriana (Triana) Cogn. } \\
\text { T. grandifolia Cogn. }\end{array}$ & $\begin{array}{l}\text { Trigonopedia sp. } \\
\text { halictids }\end{array}$ & $\begin{array}{l}\text { original } \\
\text { original }\end{array}$ \\
\hline
\end{tabular}


TABle 4. Continued.

\begin{tabular}{l}
\hline \hline \\
\hline T. grossa (L. f.) Cogn. \\
T. hospita (DC.) Cogn. \\
T. longifolia (Vahl) Baillon \\
T. moricandiana (DC.) Baillon \\
T. mutabilis (Vell.) Cogn. \\
T. pilosa Cogn. \\
T. spruceana Cogn. \\
T. trichopoda (DC.) Baillon \\
T. ursina (Cham.) Cogn.
\end{tabular}

RHEXIEAE

Rhexia virginica $\mathrm{L}$.

MICONIEAE

Bellucia acutata Pilger

B. aequiloba Pilger

B. dichotoma Cogn. (= B. im. perialis)

B. grossularioides (L.) $\mathrm{Tr}$.

B. pentamera Naudin (=B. axinanthera)

Chalybea corymbifera Naudin (= Pachyanthus corymbifera

(Naudin) Cogn.)

Clidemia bullosa DC.

C. capitellata (Bonpl.) D. Don

C. hirta (L.) D. Don

C. japurensis DC.

C. rubra (Aubl.) Mart.

Henriettea horridula Pilger

Henriettella caudata Gleason

Huilea macrocarpa Uribe

H. penduliflora Wurd.

Leandra micropetala Cogn.

L. secundiflora (DC.) Cogn.

Leandra sp. 884

Loreya riparia Renner

L. spruceana Triana bats (Anoura geoffroyi); humming. birds

halictids

halictids

Bombus morio

Bombus morio

Micrommation larocai

Augochloropsis callichroa

Bombus morio

Bombus atratus; Xylocopa augusti; Exomalopsis sp.; Augochloropsis cleopatra, A. euphrosyne, A. iris, A. leucotricha, A. scabrifrons, A. sparsilis, A. terrestris

“bees”; bumblebees

Xylocopa frontalis; Centris lilacina Xylocopa spp.; Epicharis sp. Ptilotopus superbus; Centris lilacina, Centris sp. nov.; Oxaea flavescens; Epicharis conica, E. rustica, E. affinis; Xylocopa frontalis, X. tegulata, X. similis; Bombus spp.; Euglossa intersecta, E. cf. ignita; Eulaema meriana, Eulaema nigrita, E. moczaryi

Xylocopa spp.; Centris spp.; Epicharis conica; Bombus sp.; Euglossa sp.

Xylocopa frontalis; Centris spp.; Epicharis sp.

hummingbirds

Melipona eburnea; Augochloropsis sp.

Augochloropsis sp.

Augochloropsis hebescens, A. callichroa, halictids; Melipona fulva,

M. lateralis, Melipona spp.; Euglossa sp.

Augochloropsis sp.; Melipona eburnea, M. compressipes, Melipona spp.

halictids; Melipona sp.

Xylocopa tegulata; Melipona sp.

Euglossa sp.; Melipona sp.

hummingbirds

hummingbirds

Augochloropsis sp.; Exomalopsini

Augochloropsis sp.

halictids

Xylocopa frontalis

Eulaema meriana; Xylocopa frontalis
References

Vogel, 1957

original

Renner, 1984

original

original

Laroca, 1970

Renner, 1984

Laroca, 1970

Laroca, 1970

Leggett, 1881; Eyde \& Teeri, 1967

Renner, 1984, 1986/1987

Renner, 1986/1987

Renner, 1984, 1986/1987

Roubik, 1979b; Renner, 1984, $1986 / 1987$

Renner, 1984, 1986/1987

B. Stein, pers. comm., 1986

Renner, 1984, and original

Renner, 1984

Renner, 1984, and original

Renner, 1984

Renner, 1984

Renner, 1984

Renner, 1984

Snow \& Snow, 1980

Snow \& Snow, 1980

Renner, 1984

Renner, 1984

Renner, 1984

original

Vogel, 1966; Renner, 1984, in press 
TABle 4. Continued.

\begin{tabular}{|c|c|c|}
\hline & Important Pollinators ${ }^{1}$ & References \\
\hline Miconia alata (Aubl.) DC. & $\begin{array}{l}\text { Melipona fulva; Lophothygater de- } \\
\text { corata; Paratetrapedia sp.; Au- } \\
\text { gochloropsis hebescens, A. cu- } \\
\text { preola, A. callichora; } \\
\text { Pseudaugochloropsis graminea; } \\
\text { Exomalopsis auropilosa; gen. } \\
\text { nov. near Megommation }\end{array}$ & Renner, 1984 and original \\
\hline M. alborufescens Naudin & Augochloropsis callichroa & original \\
\hline M. affinis DC. & medium-sized bees & Bawa et al., 1985 \\
\hline M. argentea $\left(\mathrm{S}_{w}\right)$ DC. & "numerous spp. of bees" & Frankie, 1976 \\
\hline M. argyrophylla DC. & halictids & Renner, 1984 \\
\hline M. dispar Benth. & $\begin{array}{l}\text { Augochloropsis sp.; Exomalopsini; } \\
\text { Melipona marginata; Xylocopa } \\
\text { similis }\end{array}$ & Renner, 1984, and original \\
\hline M. egensis Cogn. & $\begin{array}{l}\text { Xylocopa ornata; Exomalopsis au- } \\
\text { ropilosa; Paratetrapedia } \mathrm{sp.}\end{array}$ & original \\
\hline M. gratissima Benth. & Xylocopa frontalis; Melipona sp. & Renner, 1984 \\
\hline M. ibaguensis (Bonpl.) Triana & $\begin{array}{l}\text { Augochloropsis sp.; Xylocopa tegu- } \\
\text { lata, X. muscaria; Melipona sp.; } \\
\text { Exomalopsini }\end{array}$ & Renner, 1984, and original \\
\hline $\begin{array}{l}\text { M. minutiflora (Bonpl.) DC. } \\
(824)\end{array}$ & $\begin{array}{l}\text { Augochloropsis callichroa; halic- } \\
\text { tids; Melipona sp. }\end{array}$ & $\begin{array}{l}\text { Renner, 1984; Ducke, 1902; Mori } \\
\quad \text { \& Pipoly, 1984 }\end{array}$ \\
\hline M. myriantha Benth. & Melipona sp. & Renner, 1984 \\
\hline M. nervosa (Smith) Triana & $\begin{array}{l}\text { Paratetrapedia sp.; Augochloropsis } \\
\text { sp. }\end{array}$ & Renner, 1984 \\
\hline M. pileata DC. & Augochloropsis spp. & Renner, 1984 \\
\hline M. poeppigii Triana & Melipona fulva; Paratetrapedia sp. & original \\
\hline M. radulifolia (Benth.) Naudin & Paratetrapedia sp. & Renner, 1984 \\
\hline M. robinsoniana Cogn. & Xylocopa darwinii & $\begin{array}{l}\text { Linsley et al., 1966; McMullen, } \\
\quad 1985\end{array}$ \\
\hline M. rufescens (Aubl.) DC. & Melipona eburnea; Thygater sp. & Renner, 1984 \\
\hline M. splendens (Sw.) Griseb. & Melipona sp. & Renner, 1984 \\
\hline $\begin{array}{l}\text { M. tomentosa (L. C. Rich.) D. } \\
\text { Don ex DC. }\end{array}$ & $\begin{array}{l}\text { Xylocopa frontalis, X. similis; Me- } \\
\quad \text { lipona spp. }\end{array}$ & Renner, 1984 \\
\hline Miconia sp. 254 & Augochloropsis sp. & Renner, 1984 \\
\hline Miconia sp. 626 & Melipona sp. & original \\
\hline Miconia sp. 1004 & Xylocopa tegulata; Melipona fulva & original \\
\hline Miconia sp. & Eulaema cingulata & Dodson, 1966 \\
\hline Miconia sp. & Melipona sp. & Roubik, pers. comm., 1987 \\
\hline Myriaspora egensis DC. & Xylocopa sp. & original \\
\hline Tococa bullifera DC. & $\begin{array}{l}\text { Euglossa intersecta, E. piliventris, } \\
\text { E. chalybeata, E. ignita; Eulae- } \\
\text { ma meriana; Melipona fulva; } \\
\text { Paratetrapedia sp.; Megaloptid- } \\
\text { ia } \mathrm{sp} .\end{array}$ & Renner, 1984 \\
\hline T. coronata Benth. & $\begin{array}{l}\text { Euglossa sp., E. intersecta; Augo- } \\
\text { chloropsis sp. }\end{array}$ & Renner, 1984 and original \\
\hline T. guianensis Aubl. & $\begin{array}{l}\text { Melipona compressipes, Melipona } \\
\text { sp.; Xylocopa sp.; Eulaema sp.; } \\
\text { Augochloropsis sp. }\end{array}$ & Roubik, 1979b; Renner, 1984 \\
\hline T. longisepala Cogn. & $\begin{array}{l}\text { Eulaema mocsaryi, E. meriana; } \\
\text { Euglossa intersecta; Centris sp.; } \\
\text { Xylocopa frontalis; Melipona } \\
\text { fulva }\end{array}$ & Renner, 1984 and original \\
\hline T. pachystachya Wurd. & Bombus spp.; Xylocopa sp. & Renner, in press \\
\hline T. subciliata (DC.) Triana & Euglossa sp. & Renner, 1984 \\
\hline T. tepuiensis Wurd. & Bombus spp. & Renner, in press \\
\hline
\end{tabular}


TABLE 4. Continued.

Laroca, 1970

References

\begin{tabular}{l}
\hline \hline Tococa sp. \\
BLAKEEAE \\
Blakea anomala J. D. Smith \\
\\
B. austin-smithii Standley \\
B. chlorantha Almeda \\
B. gracilis Hemsley
\end{tabular}

B. penduliflora Almeda

$B$. sp. (as B. grandiflora Hemsley)

B. tuberculata J. D. Smith

Topobea brenesii Standley

\section{T. durandiana Cogn. \\ T. praecox Gleason}

\section{Memecylaceae}

Mouriri guianensis Aubl.

M. brevipes Hook.

M. myrtilloides (Sw.) Poiret

M. nervosa Pilger
Ceratina sp.; Euglossa spp.

Eulaema nigrita; Xylocopa frontalis

Euglossa imperialis, E. tridentata, Euglossa sp.; Paratetrapedia calcarata

Euglossa chalybeata, E. crassipunctata, E. purpurata, E. viridis; Eulaema mocsaryi, E. meriana; Xylocopa frontalis, $X$. similis, X. ornata; Melipona fulva, M. marginata
Melipona quinquefasciata;

Bombus ephippiatus, B. volucelM. flavipennis; Neocorymura rupa

rodents (Peromyscini, Oryzomini)

Bombus epihippiatus, B. volucelgulata, E. polychroma; Epicha ris spp.; Melipona fasciata; rodents (Peromyscini, Oryzomini) ombus epihippiatus, B. volucel lictids

Bombus epihippiatus, B. volucelloides; Eulaema seabrai; Xylocopa frontalis loides; Eulaema seabrai, E. poly. chroma; Epicharis sp.; Melipona fasciata; Xylocopa frontalis; chloropsis nigerrima

Euglossa sp.; Pseudaugochloropsis nigerrima

another species, since E. mexicana does not occur in Panama; J. Ackerman, pers. comm., 1985)

1 "Important pollinators" excludes the numerous Trigona species, which are omnipresent pollen scavengers on Melastomataceae. Note that "halictids" stands for many different species, the correct names of which cannot be ascertained at present.
Lumer \& Schoer, 1986

Lumer, 1980, 1982

Lumer, 1982

Lumer \& Schoer, 1986

Lumer, 1982

Lumer, 1982

Lumer, 1982

Lumer, 1982

Croat, 1978

Renner, 1984, and original original

Buchmann \& Buchmann, 1981

Renner, 1984, and original 
Bees gathering pollen from Melastomataceae flowers must be able to shake it out of the anthers or else pull pollen grains out through the pores with their tongues. This latter mode of exploiting melastome flowers is used by a group of Trigona bees and does not usually result in pollination because these bees are too small to contact the stigmas. Another subgroup of Trigona bees, characterized by having toothed mandibles, cuts up melastome anthers and robs their pollen (Laroca, 1970; Renner, 1983).

The method of pollen collecting used by all other bees to extract pollen from melastome anthers was first described correctly by van der Pijl (1954). When approaching a flower, in flight, the bees orient themselves with the group of stamens and the style and then straddle the stamens. They usually grasp the filaments or the bases of the anthers, quite often using their mandibles. Therefore, after having been visited, flowers often show necrotic spots on the outside of the stamens where the bees held onto them. When the bees position themselves over the androecium, their potentially pollen-bearing surface contacts the stigma even before they start exploiting the anthers. Also, they strive to establish a firm contact with as many stamens as their body size permits, going to considerable efforts in order to bundle all 8,10 , or 12 stamens. Close contact between the bee and the stamens is necessary for pollen collection by means of thorax vibrations, which are transmitted to the stamens and cause the pollen to stream out of the anther pores. Electrostatic forces due to the net positive charge of the bees and the negative charge of pollen greatly aid pollen-load adhesion (Thorp, 1979). When a pollen load, taken with forceps from a freshly captured bee, is released near this bee, it jumps back onto it (pers. obs.). The very rapid distortions of the thorax-box (with the wings at rest) are made possible by the unique contractile properties of the bees' fibrillar indirect flight muscles. Pollen collecting by this method is always accompanied by a characteristic buzzing sound and has been termed "buzzing method" (van der Pijl, 1954; Michener, 1962).

The rapid vibrations used when harvesting melastome pollen presumably produce a high temperature of the flight muscles, which may mean that this method of pollen collecting is particularly appropriate during the relatively cool early morning hours. Also, there are ecological constraints on the activity of dark-colored bees during the hottest hours. Buzz pollination continues with very high humidity of the air and even during slight rains.

In most melastomes, pollen deposition and re- ception is on the lower abdomen (sternotribic) or lateral on the bees (pleurotribic); it is on the back (nototribic) in a few species with downwards-curved styles (e.g., species of Meriania, Adelobotrys, Salpinga) and in Desmoscelis villosa. In these cases, bees assume quite unexpected positions in order to vibrate the stamens; in Adelobotrys rachidotricha, for instance, they buzz with the head pointing outward and downward. Relatively large flowers, such as those of Blakea, Topobea, and Bellucia, are exploited by a wide range of bees (Lumer, 1982; Renner, 1986/1987). For example, stamens of Bellucia are vibrated by 33 -mm-long Centris and 8-mm-long Augochloropsis bees. In the case of self-compatible species, visits by bees too small to contact the stigma and deposit foreign pollen may still result in self-pollen landing on the stigma when such small bees vibrate one or a few stamens.

Bees visiting Melastomataceae exhibit considerable steadiness during individual foraging flights: pollen loads often consist of almost pure melastome pollen with very few grains from nectariferous flowers or with grains from Cassia mixed in (Renner, 1984). Cassia has buzz-pollinated flowers, and bees often visit several such flowers (see below).

In Brazilian Amazonia, I have captured 80 species of bees, including the Africanized honey bee, Apis mellifera scutellata Lepeletier, vibrating melastomes. (A list of all bees, including the species of Trigona, may be obtained upon request.) Additional species have been captured in extra-Amazonian areas and at higher altitudes. To date, at least 100 species of bees have been found pollinating melastome flowers (Table 4; this does not include the numerous species of pollen-scavenging Trigona bees). The relatively few groups of bees that have been successful in tropical forest environments, the euglossines, halictids, Xylocopa, and Melipona (Michener, 1979) are the most important pollinators of lowland melastomes. In savannas, Centridini, Oxaeidae, Exomalopsini, and Ptiloglossa (Colletidae) become important, as do bumblebees in southern Brazil and at higher altitudes. The bee fauna in neotropical forests and savannas is still incompletely known; however, based on bee inventories in Panama, Costa Rica, French Guiana, Surinam, and at the mouth of the Amazon (Heithaus, 1979; Roubik, 1979a, b; and ref. in Michener, 1979), it may be estimated that only about 300 species of bees occur in these forests, a number that includes non-flower-visiting parasitic and leaf-cutter bees. Thus, between 80 and 100 species collecting pollen from Melastomataceae represents a high proportion of the total Amazonian bee fauna. 


\section{b) Floral attractants}

Melastome pollen is an important pollen source for neotropical bees. This is clear from a survey of literature on bee foraging: it is collected by female euglossines (Moure, 1970; Janzen, 1971; Michener et al., 1978; Roubik, 1979a, b; Dressler, 1982; Renner, 1984), meliponines and halictids (Ducke, 1901, 1902; Laroca, 1970; Roubik, 1979a, b; Michener et al., 1978; Absy \& Kerr, 1977; Absy et al., 1980), mixed with Malpighiaceae oil by anthophorine bees (Vogel, 1974; Renner, 1984), and makes up 96-100\% of the nest provision of the colletid bee Crawfordapis (Roubik \& Michener, 1985). A chemical analysis of Bellucia dichotoma pollen revealed high nitrogen and total crude protein. (I am indebted to S. Buchmann for analyzing a pollen sample in 1983.) In this species, the pollen has a strong perfume resembling that of the flowers.

Secondary attractants for bees include sweet or perfumelike floral odors produced by the petals, the connective, and the upper section of the style (as determined by neutral red staining; pers. obs.; method, Vogel, 1963), and the presence of a landing platform provided by the androecium. I have never seen bees land on the petals. Frequently, the filaments are adorned with glandular or eglandular hairs, which may make them easier for the bees to grasp. Also, direct alighting on the stamens may make visits to flowers with poricidal anthers particularly rewarding compared with flowers requiring further crawling and searching after landing.

Most Melastomataceae seem to be visited and pollinated early in the morning, sometimes $20 \mathrm{~min}$ utes before sunrise (F. Mueller, 1873; Frankie, 1976; Lumer, 1982; Renner, 1984), and many species close their petals by late morning. However, flowers of Monochaetum and Rhynchanthera open late, two to four hours after sunrise (Almeda, 1978; pers. obs.). Shedding of the flower parts usually starts at the end of the second day. Again, Monochaetum differs in having flowers that stay open for three days (Almeda, 1978), and I have seen Graffenrieda fruticosa flowers staying attractive for at least seven days. In the latter case, the adverse climatic conditions at $2,200 \mathrm{~m}$ altitude, where Graffenrieda fruticosa occurs, may place a high selective advantage on longer flowering periods which presumably heighten the flowers' chances of being visited.

The typical bee colors of most Melastomataceae flowers, white, purple, or pink, are due to total reflection and anthocyanins (Lowry, 1976; Bobbio et al., 1985). Yellow and orange flowers evolved repeatedly in isolated species throughout the fam- ily. Contrasting marks at the base of the petals and color changes during anthesis are frequent in Melastomataceae (F. Mueller, 1873; Ule, 1896; Wurdack, 1980; Renner, 1984). The colors change from white to red, from yellow to red, from white to purple, or from pink to white. The bright white of the small flowers of many herbaceous understory melastomes, e.g., species of Aciotis, Leandra, Clidemia, Miconia, and Ossaea, may be of selective advantage under conditions of low light intensity. Some melastome flowers tested (using a Kodak 18A Wratten filter) did not absorb ultraviolet light (Lumer, 1982), as was to be expected from the absence of flavonols from their petals.

The androecia of many species in the capsularfruited tribes Merianieae, Bertolonieae, Microlicieae, Tibouchineae, and Rhexieae show a strong morphological differentiation into two sets of stamens. In addition, in such flowers the connective appendages may be large and stiff, forming 5, 10, or 15 usually yellow spurs, sometimes positioned like true anthers. Based on his brother's (Fritz Mueller) observations on a southern Brazilian species of "Heeria," Hermann Mueller (1881, 1883) was the first to suggest that this dimorphism was the ultimate result of a "division of labor" between the two sets of stamens (his illustration shows that his brother was probably dealing with a species of Microlicia; Heeria is synonymous with Heterocentron, which does not occur in southern Brazil). According to this idea, the conspicuous anthers attract the bees and offer "food pollen" as a reward, whereas the less conspicuous ones contain "functional pollen." The "functional pollen" is supposed to be placed on a part of the bee where it will not be groomed off and used as food for larvae. It will instead function in fertilization. Hermann Mueller's idea caught on immediately (Forbes, 1882; F. Mueller, 1883) and is usually mentioned as typical of the pollination strategy of Melastomataceae (e.g., Cammerloher, 1931; Percival, 1965; Kugler, 1970; Proctor \& Yeo, 1972; Baker, 1978; Faegri \& van der Pijl, 1979).

A partitioning of the bee surface would be necessary for selection to favor the pollen grains from one set of stamens over those from the other and thus lead to pollen dimorphism. Pollination mechanisms involving vibration of anthers, however, may not allow a sufficiently precise placement of pollen from two sets of stamens on bees. Forbes (1882), working with unnamed species of Melastoma, reported that "only the pollen from the long stamens seemed [sic] to be fertile"; yet he found pollen from both types of stamens on the stigmas. To date, with the exception of Forbes's report 
(1882), no morphological or physiological differences between pollen grains from dimorphic stamens have been found in any of the Melastomataceae studied (Darwin, 1876; Ziegler, 1925; Whiffin, 1973; Almeda, 1977; Renner, 1984). Further experimental fieldwork is needed to assess whether the staminal dimorphism and the stamen appendages have a function beyond that of enhancing the visual attractiveness of the flowers and making the stamens easier to grasp for bees. In view of the importance of pollen aromas for guiding bee behavior (cf. Dobson, 1987), it is uncertain if the visual cues provided by the yellow connective appendages by themselves elicit pollen-collecting behavior.

POLLINATION OF NECTAR-PRODUCING MELASTOMATACEAE BY BIRDS, BATS, RODENTS, AND BEES

Some 60 species of Meriania, Centronia, Brachyotum, Tibouchina, Miconia, Huilaea, Chalybea, and Blakea, all of them, except the species of Miconia, occurring at higher altitudes $(1,600-3,400 \mathrm{~m})$, offer nectar as a reward for their pollinators. On the basis of their character syndrome (see below), Meriania drakei (Cogn.) Wurd., M. furvanthera Wurd., Axinaea weberbaueri Cogn., and several other species of Meriania, Axinaea, Centronia, and Tibouchina are expected to produce nectar, too.

The first reports on nectar production in melastomes are by Ule $(1895,1896)$. In three species of Tibouchina (Table 4) growing on the Brazilian Itatiaia mountain range, he observed that nectar was produced by the staminal vascular bundle and secreted either directly from the filaments or sometimes through a slit in the filaments. He suggested bumblebees as the probable pollinators of these species. At Ule's request, the chemist T. Peckolt analyzed the stamens of another species that did not secrete nectar (Ule gave the name as $T$. glareosa Cogn.; there is no such name, and he probably meant T. granulosa Cogn.). Peckolt found that sugar was present "in great quantity," which suggested to Ule (1896) that this was likely true for other species of Tibouchina as well. In several other nectariferous melastomes, slits in the upper ventral side of the filaments have been observed, e.g., in Tibouchina cleistoflora Ule (Ule, 1896; pers. obs.), T. grossa, Blakea chlorantha (Tobe et al., in prep.), Brachyotum ledifolium, B. andreanum Cogn., B. benthamianum Triana, and B. rugosum Wurd. (Lagerheim, 1899; pers. obs.). They have also been found in non-nectariferous species-such as Centradenia grandiflora, Heterocentrum roseum, Triolena pustulata (as Bertolonia pubescens) - and in paleotropical species of Dissotis and Medinilla (Ziegler, 1925). These slits appear not to be consistently formed sutures, but rather spontaneous ruptures. The anatomy of the nectariferous tissue is being studied by Tobe et al. (in prep.).

Reports of nectar in Miconia minutiflora (Mori \& Pipoly, 1984) are unclear, since its presence in the minute flowers $(2.5 \mathrm{~mm}$ in diameter, style 4 mm long) was only presumed. In central Amazonia, where I have observed this species, it did not produce nectar (Renner, 1984). However, in another Miconia species in the Belém region at the mouth of the Amazon, Roubik measured floral nectar of ca. 20\% sugar concentration and collected Melipona and Apis (!) bees (Roubik, pers. comm. 1987). Recently, nectar production has also been confirmed in the Antillean endemics Miconia sintenisii Cogn. and Mecranium amygdalinum (Desr.) W right (Dent, pers. comm.). Miconia robinsoniana, also with minute white flowers, was included under "nectar sources" in a summary of flower records for Xylocopa darwinii by Linsley et al. (1966). McMullen, who made further observations on the floral visits of this bee on Galápagos (McMullen, 1985), has never noticed any nectar in M. robinsoniana (McMullen, pers. comm., 1987).

Except in Miconia, nectar production in Melastomataceae is correlated with floral morpholog. ical changes that relate to the fact that the nectar collectors do not vibrate the stamens. Therefore, when nectar instead of pollen is the pollinator reward, the latter must be deposited on a visitor during nectar uptake. This is achieved by the anthers having rather large pores and being stiffened, particularly at the base. Pressure on the base of the anthers exerted by the head of a bee, the bill of a bird, or the front paws of rodents clasping the flowers ejects pollen onto the visitor. Lagerheim (1899) therefore speaks of "bellow" anthers; he found that a match the thickness of a hummingbird bill pushed into the flower of Brachyotum ledifolium so that it pressed the anther bases brought forth a pollen cloud $3 \mathrm{~cm}$ long. The flowers of the nectariferous species are inclined or pendent and, in the case of the bird-pollinated ones, usually dark red, deep purple, or bluish to almost black. In Brachyotum ledifolium the petals are yellow, but the hypanthium is covered with bright red hairs. In nectariferous melastomes, the petals do not spread at anthesis but remain convolute almost like in bud. Vogel (1957) reported a nocturnal cabbage odor in the predominantly bat-pollinated Tibouchina grossa. The other nectariferous melastomes are reported as scentless. 
The bulk of nectariferous melastomes seem hummingbird-pollinated (Table 4). Most of them belong to the genus Brachyotum, which comprises 50 species, the majority ornithophilous (Wurdack, 1965, 1980; Lagerheim, 1899; Vogel, 1957; Fitzpatrick et al., 1979; label data). Other bird-pollinated species are Meriania tomentosa (Cogn.) Wurd. (Centronia excelsa (Bonpl.) DC.; van der Werff, pers. comm.; D. Neill, pers. comm.). Nectariferous and hummingbird-pollinated species in the Miconieae are Huilea macrocarpa, H. penduliflora (Snow \& Snow, 1980) and probably also Chalybea corymbifera (in which Stein observed nectar but no pollination events; pers. comm., 1987). The Colombian Tibouchina grossa is pollinated by bats and hummingbirds (Vogel, 1957; Stein, pers. comm., 1987) and certainly the same is true of T. Stricta Wurd. and T. reticulata Cogn.

New World rats and mice of the tribes Peromycini and Oryzomini pollinate at least three nectariferous species of Blakea in Costa Rica (Lumer, 1980, 1982; Lumer \& Schoer, 1986). Lumer found that bees and hummingbirds rarely visited the flowers of these species during the day but that nectar secretion was nocturnal. The Blakea species involved are hemiepiphytic, and their flowers are positioned close to the stem where the rodents can reach them conveniently.

The nectar of Blakea chlorantha contains ca. $11 \%$ sucrose (Lumer, 1982, and pers. comm.); that of Huilea macrocarpa has $12-16 \%$ with a mean of $13.4 \%$ (Snow \& Snow, 1980); that of Chalybea corymbifera $16.5 \%$; and that of Brachyotum ledifolium 20-22\% (Tobe et al., in prep.). It would thus be classified as relatively rich in sucrose in the nectar class system of Baker \& Baker (1983), who found that sucrose-rich or sucrose-dominated nectar prevails in hummingbird flowers. Thus, nectariferous melastomes offer a reward "intended" for pollinators other than bees.

The evolutionary origin of pollination systems involving nectar in Melastomataceae is considered below.

\section{MEMECYLACEAE}

In their pollination mechanism, the neotropical Memecylaceae closely resemble the Melastomataceae, and the above description of bees' methods of pollen collecting from melastome flowers also applies to them. In Mouriri, the anthers are stiff, with thickened endothecial walls, and are slightly prolonged just above the insertion of the filaments. The prolongations, termed "caudae" (Morley, 1953), are used as footholds by the bees exploiting
Mouriri anthers. Pollination in four species of Mouriri has been observed (Table 4); the main pollinators seem to be euglossines, followed by Xyloco$p a$ and Melipona species. They are legitimate visitors, i.e., large enough to contact the stigma regularly while collecting the reward. Memecylaceae anthers differ from those of Melastomataceae in bearing dorsal concave glands, $0.3-0.8 \mathrm{~mm}$ long. (For illustrations of the permutations in size and position of these glands in Mouriri and $\mathrm{Vo}_{\mathrm{o}}$ tomita, see Morley, 1953, 1976, and 1985.) None of the legitimate pollinators show any interest in the staminal glands (Buchmann \& Buchmann, 1981; Renner, 1984, and unpubl.).

Earlier (1984), I reported that Melipona marginata and $M$. fulva sometimes collected the secretion from the glands with the front legs, and that they mixed this substance with their pollen loads between pollen-collecting bouts on the wing. Further field observations, however, have convinced me that Melipona does not regularly touch the glandular surface when buzzing. The bees grip the stamens by the anther caudas just below the glands, and it is difficult to see what they do with their feet or their mouth parts when vibrating because they curve tightly over the anthers to allow the pollen stream to settle on their bodies. Between buzzing bouts, Melipona bees (and the euglossines) rotate over the androecium so as to drum out the pollen from all ten anthers. Staining of their freshly collected corbicular loads with Fehling solution showed that the pollen had been wetted with sugar. With Sudan III (a lipid stain), Mouriri pollen grains stained red, but the secretion holding them together did not; this implies that the pollen contains lipids, whereas the secretion did not.

Mouriri flowers are also visited by several species of Trigona. These small bees do not regularly contact the stigma but rob pollen from the anthers, which they are unable to buzz. Their role as pollen scavengers and robbers in Melastomataceae is well known (Renner, 1983). When cutting up anthers of Mouriri nervosa to reach pollen grains further down, Trigona williana very rarely touched the glands (Renner, 1984, and unpubl.). Most often, T. williana cut off the anthers above the glands, then stopped and started cutting and robbing the pollen of another anther. This shows that this pollen robber, like the legitimate pollinators, does not regularly collect the glandular exudates. Buchmann \& Buchmann (1981) have observed T. pallens manipulating the glands of $M$. myrtilloides with the front legs and mouth parts.

The secretion of the staminal glands is yellow, viscous, stains red in Sudan III, and tests positive 
for fatty substances. That of the relatively large (0.8 $\mathrm{mm}$ long) glands of $M$. lunatanthera forms myelin figures in water after a drop of sodium hydroxide is added (pers. obs.; method from Vogel, 1974). The secretion in M. myrtilloides has been reported to be a lipoidal mixture of many compounds (e.g., phenolic compounds, small amounts of glucose and saponin, at least 13 fatty acids, and free fatty acids) and to be "extremely rich in nonlipid constituents" (Buchmann \& Buchmann, 1981).

Since the secretions from the floral glands contain lipids, the relationship between Mouriri and oil-collecting bees is of particular interest. Floral oils are collected by bees belonging to relatively few genera of Anthophoridae (e.g., Centris, Epicharis, Monoeca, Paratetrapedia, Tapinotaspis, Tetrapedia; cf. Vogel, 1974; Neff \& Simpson, 1981). Although many of these collect pollen from Melastomataceae (comp. Table 4), to date only a single anthophorid bee species, Paratetrapedia calcarata, has been observed on Mouriri (Buchmann \& Buchmann, 1981). This bee, due to its small size $(7-8 \mathrm{~mm}$ long), rarely vibrated more than two or three of the ten anthers in each flower and only rarely touched the stigma (l.c.). Also, Buchmann \& Buchmann (1981) reported that Paratetrapedia calcarata was an infrequent visitor of M. myrtilloides ( 16 times during 50 hours) and not a legitimate pollinator.

The secretion of the staminal glands forms a shiny film on the gland cuticle and in this respect differs greatly from the lipid-filled blisters of other epithelial elaiophores (Vogel, 1974). Paratetrapedia has scrapers on the fore-basitarsi (Neff \& Simpson, 1981) and ruptures the cuticle of the elaiophores from which it collects oils. While scrutinizing hundreds of stamens from Mouriri nervosa and $M$. guianensis under the stereoscope in order to collect their glandular secretions, I never found signs of the glands having been scraped. This was perhaps to be expected, since the secretion forms a film on the outside of the cuticle.

A further problem in understanding the role of the staminal glands is that they are so small and positioned in such a way that the collection of any exudate either with the fore-basitarsi or the mouth parts by the medium-sized bees that pollinate $\mathrm{Mou}$ riri is difficult to envisage. Visitors small enough to be attracted by the minute quantities of secretion offered (such as the Paratetrapedia species observed by Buchmann \& Buchmann, 1981) are not large enough to touch the stigmatic surface when manipulating the glands, especially since the styles in Mouriri are always longer than the stamens and the stigmas are held well away from the anthers.
A possible role of the staminal glands suggested by Morley (1976) is odor production. Mouriri species often have a strong perfume scent (Buchmann \& Buchmann, 1981; Renner, 1984). Perhaps a volatile oil would persist longer when mixed with the fatty oils and other compounds. Buchmann \& Buchmann (l.c.) suggested that the glands, which absorb ultraviolet light, might visually guide the bees to the pollen. Extending this idea, one might ascribe a pseudonectary role to the staminal glands. It is interesting that in the Bonnetiaceae, at least 23 species of Caraipa and Mahurea have very similar minute urceolate glands positioned subapically on the connective (Kubitzki, 1978). The role of these glands, as of those of Mouriri, is obscure.

\section{Seed Dispersal in Melastomataceae}

Of the neotropical Melastomataceae, $40 \%$ have capsular fruits and wind-dispersed seeds (Renner, 1986). The remaining $60 \%$ have soft, juicy berries and endozoochorous seeds. Animal-dispersed Melastomataceae are most diverse in lowlands and montane forests, whereas the capsular-fruited tribes are richest in species in various types of savannas. Melastome capsules are often sturdy and persist on the plants for many months, during which the seeds are gradually shaken out. There are no data on dispersal distances in the wind-dispersed species; however, the many striking disjunctions observed in savanna melastomes (Renner, 1987b, and in press) probably attest to their good dispersability. Small herbaceous understory species with broadly conical capsules (Salpinga, Monolena, Bertolo. nia) may also have their seeds dispersed ballistically by raindrops.

Melastome berries range in size from five to ca. $35 \mathrm{~mm}$ in diameter. The smaller ones are of out standing importance for small frugivorous birds throughout the Neotropics (Huber, 1910; Snow, 1965, 1971, 1981; Ricklefs, 1977; Skutch, 1980; Lumer, 1982; Wheelwright et al., 1984; R. Bierregård, pers. comm., and many additional references). Epiphytic Melastomataceae are mostly birddispersed (Renner, 1986), as are many of the most successful pioneer species, for example, Clidemia hirta, which has become a noxious weed in Africa, Asia, and Hawaii.

Fruit attributes, such as position, size, aroma, and color, indicate that different animal classes act as the principal dispersers in different species groups. For example, black spider monkeys are the predominant consumers and likely dispersers of the green, slightly astringent and relatively large berries of the tree Bellucia grossularioides in Surinam (Roosmalen, pers. comm.). In French Guiana, 
this species is a favorite with the Philander opossum (Atramentowicz, 1982), which also eats Henriettea and Loreya fruits. However, a search of recent literature on feeding records (Renner, 1987a) showed that Melastomataceae are not among the preferred foods of either monkeys or bats (for bats, Fleming, 1986; but compare Foresta et al., 1984, and Fleming, 1988). Perhaps because the majority of Melastomataceae are shrubs or understory trees with terminally positioned infructescences, they are unsuited for foraging monkeys and bats. Also, the taste of the mostly sweet, watery melastome berries may not be to the liking of monkeys, which apparently prefer slightly acidic fruits (Sourd \& Gautier-Hion, 1986).

Fruits that have fallen to the ground are often eaten by turtles (Moskowitz, pers. comm.; Roosmalen, pers. comm.), lizards (W. Magnusson, pers. comm.), rodents (Magnusson \& Sanaiotti, 1987), tapirs (Huber, 1910), and ants (pers. obs.).

\section{Seed Dispersal in Memecylaceae}

All Memecylaceae have berries, ranging in size from 0.5 to $10 \mathrm{~cm}$ long; color at maturity is most commonly yellow to red, less often it is purpleblack to black (Morley, 1976). The smaller-fruited species are surely bird dispersed; some larger-fruited ones are monkey-dispersed (Roosmalen, 1985). Fish eat the fruits of at least one riverine Mouriri species (Goulding, 1980), which is curious, since Morley (1976) reported that two other species are used for poisoning fish.

\section{Reproductive Biology and the Evolution of Melastomataceae and Memecylaceae}

Floral morphology in Melastomataceae and Memecylaceae can only be understood as the result of coevolution between the earliest ancestors of these groups and pollen-collecting bees. Flowers of the Old World members of both families have the same basic construction as those of their New World relatives and, although little information is available on their floral biology, van der Pijl's (1939) detailed description of the pollination mechanism in some species of Osbeckia, Medinilla, and Melastoma makes it likely that they are pollinated in the same manner as the New World ones. The pollen offered in the poricidal stamens of both families is accessible exclusively to bees, but not to many of the most common ones, such as the numerous species of Trigona and the paleotropical Apis, which apparently do not vibrate stamens. Most Melastomataceae and Memecylaceae have "modern" pollen flowers in the sense of Vogel (1978), in which the visitor is forced into a fixed position while harvesting the reward and in which the position of the stigma is of relatively little consequence because of the deposition of pollen on a large part of the visitor's surface.

There appears to be little opportunity for partitioning the bee body. Sharing of pollinators, noted rather frequently among sympatric buzz flowers (Linsley \& Cazier, 1963; Macior, 1970, 1971; Thorp \& Estes, 1975; Lumer, 1982; Renner, 1984), results in hybridization in the absence of genetic barriers. This is consistent with the fact that systematists working with Melastomataceae and Memecylaceae often notice hybrids (Kral \& Bostick, 1969; Whiffin, 1973; Morley, 1976; Almeda, 1978; Solt \& Wurdack, 1980; Renner, in press). The data on Melastomataceae breeding systems corroborate the suggestion that apomixis may be more widespread in the tropics than formerly thought (Kaur et al., 1978; Ashton, pers. comm., 1987). Apomictic seed production would make such species independent of pollinators, while retaining the advantages of producing seeds, i.e., the potential for dispersal and dormancy. Most notably, it would give them the capacity to build up a new population from a single propagule.

Statements that "details of the structure of the anthers doubtless relate to mechanisms of pollination" (Cronquist, 1981) or "in a family, such as the Melastomataceae, where floral evolution has been extensive, one may logically conclude that the diverse floral types represent adaptations to different pollination systems" (Almeda, 1977) are not corroborated by field data. Rather, Melastomataceae seem to confirm Macior's (1971) suggestion that buzz pollination is so successful a system that, once it is established, hardly any other pollination mechanism evolves, even in species-rich groups. Compared with other families, such as Lecythidaceae, Gesneriaceae, and Bignoniaceae, for which the overall pollination spectrum is fairly well known, Melastomataceae and Memecylaceae show little diversification in floral morphology and pollination strategies. In this they resemble Malpighiaceae (Anderson, 1979) and Solanaceae (Symon, 1979), which also have relatively specialized pollination systems and faithful bee pollinators. Such lack of diversification could be interpreted as the result of being stuck on an adaptive peak.

Melastomataceae that offer nectar are pollinated by a broader range of pollinator classes, namely by hummingbirds, bats, rodents, and bees; this statement applies to the individual species as well, several of which have more than one important pollinator type, for instance birds and bats in Tibouchina grossa and bees and rodents in Blakea chlorantha. Cruden (1972) suggested, as a possible 
ecological significance for nectar production at higher altitudes, that under adverse climatic conditions the homoiothermic birds and mammals may be more reliable pollinators than bees. If valid, this explanation would apply to all now known nectarproducing Melastomataceae, except the species of Miconia.

Whether the thousands of pollen-only Melastomataceae derived from ancestors with nectar flowers or whether the proto-Melastomataceae never possessed nectaries (as suggested by Vogel, 1978) is a difficult question. Phylogenetically, the extant nectariferous species belong to groups not closely related: Meriania and Centronia are in the capsular-fruited Merianieae (as is Axinaea, which probably also has some nectariferous species, see above); Brachyotum and Tibouchina are in the likewise capsular-fruited Tibouchineae; Chalybea, Huilea, and Miconia are in the berry-fruited Miconieae; and Blakea is in the also berry-fruited Blakeeae. Thus nectar production occurs in at least five evolutionary lines: the first leading to Meriania and Centronia (which are not closely related to each other), the second to Brachyotum and Tibouchina (the first genus is a segregate of the second; Wurdack, 1954), the third to Chalybea and Huilea (two intimately related genera), the fourth to Miconia (with 1,000 species, only two of which to date are securely known to produce nectar), and the fifth to Blakea. Nectaries could have originated independently in all these lineages.

The problem is confounded by the slits observed in the filaments of some non-nectariferous Melastomataceae exactly in the same position as in some of the nectariferous species, where nectar is secreted through them. They occur in species of Heterocentron (Tibouchineae), Bertolonia (Bertolonieae), and Centradenia (Microlicieae) and in the paleotropical genera Medinilla and Dissotis (compare above under nectariferous melastomes) and may be rudimentary, nonfunctional nectaries. Thus, to date, only in two of the neotropical tribesthe small Rhexieae with 64 species and the Cyphostyleae with nine species - are nectar production or presumed rudimentary nectaries absent. In view of the presence of nectaries in at least five neotropical lineages and the indications of their former presence in two further ones, their parallel independent origin becomes hard to accept. Instead, I suggest that the capacity for developing nectaries is basic in the Melastomataceae but suppressed in most modern members.

To understand the origin of the memecyloid connective gland, whatever the chemical composition of its secretion, the following observations seem relevant. Functioning nectaries have been observed on the hypanthia of Memecylon edule Roxb. (= M. ramiflorum Desr.; Burck, 1891) and M. floribundum Blume (Zimmermann, 1932). Also, I have frequently seen ants on the hypanthia of Mouriri nervosa, which may be an indication that this neotropical Memecylaceae also secretes sugar, albeit in minute quantities. Evolutionarily, the capacity to secrete minute quantities of exudates directly through the hypanthium epidermis, without conspicuous morphological structures, may be correlated with the development of the staminal glands. However, data on the pollinators and the chemical composition of the exudates of paleotropical Memecylaceae are needed before the significance of the staminal glands in the family as a whole can be assessed. The Memecylaceae have ca. 340 Old World and 89 New World species, and it should be noted that, in the opinion of Morley (1953), the New World Memecylaceae are more basal than the Old World ones.

There is a great amount of parallelism between the Old World and the New World tribes in the Melastomataceae: the Oxysporeae, Sonerileae, Dissochaeteae, and Osbeckieae resemble more or less closely the Merianieae, Bertolonieae, Miconieae, and Tibouchineae, respectively, of the New World (Gleason, 1932). Based on wood anatomy, the differentiation into at least some of these tribes and into Melastomataceae and Memecylaceae occurred before the breakup of Pangaea (Vliet et al., 1981). However, the coevolutionary relationships between bees and proto-Melastomataceae, which are a conditio sine qua non for the evolution of the melastome androecium, can only have begun during the mid-Cretaceous when bees arose (Michener, 1979). The mid-Cretaceous may thus have been the time when the ancestors of these families switched from nectar-reward flowers to pollen-reward flowers (with the pollen presented in poricidal anthers and available only to bees). Likely, the Melastomataceae and Memecylaceae originated during Aptian times (lower mid-Cretaceous, 110 million years before present), when the gap between Brazil and Ghana was still very narrow; their east and west Gondwanian lineages, probably coevolved in parallel with the earliest bees. The oldest fossil melastome leaves are known from the early Eocene (Hickey, 1977), about 53 million years before present, and Eocene and Miocene leaves occur widely scattered throughout the Northern Hemisphere (Wurdack \& Kral, 1982). Therefore, members of the family (and some of their pollinators?) at one time may have dispersed via Europe between North America and Africa.

The Melastomataceae, with two-thirds of their species in the Neotropics, have much of their di- 
versity centered in areas of varied topographythe Andes, Guayana, and south-central Brazil. The neotropical Memecylaceae, on the other hand, are centered in the Amazonian lowlands, where they appear adapted to different soil types (pers. obs.). The very early shift from anemochory to zoochory in the Melastomataceae was probably one of the reasons for their success in the understory of closed forests, where animal dispersal is more advantageous than wind dispersal. (The capsular-fruited tribes are much more diverse in open habitats and have relatively few species in forests.) The crucial points in the radiation of large groups within the Melastomataceae, besides their different seed-dispersal strategies, have been the acquisition of particular plant architectural, indument, and leaf venation characters. Because adaptations to different pollinator types played such a limited role in the radiation within the two families, the floral biological data cannot be used to support either of the two suggested phylogenies for the Melastomataceae (Wurdack in Welle \& Koek-Noorman, 1981; Vliet et al., 1981).

\section{Literature Cited}

ABsy, M. L. \& W. E. KERR. 1977. Algumas plantas visitadas para obtenção de pólen por operárias de Melipona seminigra merrillae em Manaus. Acta Amazonica 7: 309-315.

—, E. B. Bezerra \& W. E. Kerr. 1980. Plantas nectaríferas utilizadas por duas espécies de Melipona da Amazônia. Acta Amazonica 10: 271-281.

AlmedA, F. 1977. Systematics of the neotropical genus Centradenia (Melastomataceae). J. Arnold Arbor. 85: 73-108.

- 1978. Systematics of the genus Monochaetum (Melastomataceae) in Mexico and Central America. Univ. Calif. Publ. Bot. 75: 1-134.

Anderson, W. R. 1979. Floral conservatism in neotropical Malpighiaceae. Biotropica 11: 219-223.

Arroyo, M. T. K. \& E. Cabrera. 1977. Preliminary self-incompatibility test for some tropical cloud forest species in Venezuela. Incomp. Newsletter 8: 72-76.

Atramentowicz, M. 1982. Influence du milieu sur l'activité locomotrice et la reproduction de Caluromys philander (L.). Rev. Ecol. (Terre Vie) 36: 373395.

BAKER, H. 1978. Chemical aspects of the pollination biology of woody plants in the tropics. Pp. 57-82 in P. B. Tomlinson \& M. H. Zimmermann (editors), Tropical Trees as Living Systems. Cambridge Univ. Press, Cambridge, London, New York, Melbourne. \& I. BAKER. 1983. Floral nectar sugar constituents in relation to pollinator type. Pp. 117-141 in C. E. Jones \& R. J. Little (editors), Handbook of Experimental Pollination Biology. S \& AE Scientific and Academic Editions, New York.

Bawa, K. S., S. H. Bullock, D. R. Perry, R. F. Coville \& M. H. Grayum. 1985. Reproductive biology of tropical lowland rain forest trees. II. Pollination systems. Amer. J. Bot. 72:346-356.

Bobbio, F. O., P. A. Bobbio \& C. H. Degaspari. 1985.
Anthocyanins from Tibouchina grandiflora. Food Chem. 18: 153-160.

Bremer, K. 1982. Lijndenia, a re-established paleotropical genus of the Melastomataceae-Memecylaceae. Nord. J. Bot. 2: 121-124.

- 1983. Taxonomy of Memecylon (Melastomataceae) in Borneo. Opera Bot. 69: 1-47.

Buchmann, S. L. \& M. D. Buchmann. 1981. Anthecology of Mouriri myrtilloides (Melastomataceae: Memecyleae), an oil flower in Panama. Biotropica 13 (suppl.): 7-24.

Burck, W. 1891. Beitraege zur Kenntnis der myrmekophilen Pflanzen und der Bedeutung der extranuptialen Nektarien. Ann. Jard. Bot. Buitenzorg 10: $75-144$.

Cammerloher, H. 1931. Bluetenbiologie I. Borntraeger Vlg., Berlin.

Candolle, A. P. DE. 1828. Melastomataceae. Prodromus Systematis Naturalis Regni Vegetabilis 3: 99202.

Cogniaux, A. 1891. Melastomaceae. In: A. \& C. de Candolle, Monographie Phanerogamarum 7: 1-1256.

Cremers, G. 1983. Architecture Végétative et Structure Inflorescentielle de Quelque Melastomataceae Guyanaises. Univ. Strasbourg, France.

Croat, T. B. 1978. Flora of Barro Colorado Island. Stanford Univ. Press, Stanford, California.

Cronquist, A. 1981. An Integrated System of Classification of Flowering Plants. Columbia Univ. Press, New York.

Cruden, R. W. 1972. Pollinators in high-elevation ecosystems: relative effectiveness of birds and bees. Science 176: 1439-1440.

Dahlgren, R. \& R. F. Thorne. 1984. The order Myrtales: circumscription, variation, and relationships. Ann. Missouri Bot. Gard. 71: 633-699.

DARwin, C. 1876. The Effects of Cross- and Self-Fertilisation in the Vegetable Kingdom. John Murray, London.

DARWIN, F. (editor). 1903. More Letters of Charles Darwin, Volume II. D. Appleton \& Comp., New York.

Dobson, H. E. M. 1987. Role of flower and pollen aromas in host-plant recognition by solitary bees. Oecologia 72: 618-623.

Dodson, C. H. 1966. Ethology of some bees of the tribe Euglossini. J. Kansas Entomol. Soc. 39: 607629.

Don, D. 1823. An illustration of the natural family of plants called Melastomaceae. Mem. Wernerian Nat. Hist. Soc. 4: 276-329.

Dressler, R. L. 1982. Biology of the orchid bees (Euglossini). Ann. Rev. Ecol. Syst. 13: 373-394.

DuCKE, A. 1901. Beobachtungen ueber Bluetenbesuch, Erscheinungszeit etc. der bei Para vorkommenden Bienen. Z. Syst. Hymenop. Dipterol. 1: 25-32, 4967.

- 1902. Beobachtungen ueber Bluetenbesuch, Erscheinungszeit etc. der bei Para vorkommenden Bienen. Allg. Z. Entomol. 7: 321-326, 360-368, 400-405, 417-422.

Etheridge, A. L. \& J. M. Herr. 1968. The development of the ovule and megagametophyte in Rhexia mariana. Canad. J. Bot. 46: 133-139.

Eyde, R. H. \& J. A. TEERI. 1967. Floral antomy of Rhexia virginica (Melastomataceae). Rhodora 69: $163-178$.

Faegri, K. \& L. van der Pijl. 1979. The Principles 
of Pollination Ecology, 3rd revised edition. Pergamon Press, Oxford, New York.

Fitzpatrick, J. W., D. E. Willard \& J. W. Terborgh. 1979. A new species of hummingbird from Peru. Wilson Bull. 91: 177-186.

Fleming, T. H. 1986. Opportunism versus specialization: the evolution of feeding in frugivorous bats. $\mathrm{Pp}$. 105-118 in A. Estrada \& T. H. Fleming (editors), Frugivores and Seed Dispersal. Junk, Dordrecht.

- 1988. The short-tailed fruit bat. Univ. Chicago Press, Chicago and London.

Forbes, H. O. 1882. Two kinds of stamens with different functions in the same flower. Nature 26: 386.

Foresta, H. DE, P. Charles-Dominioue, C. Erard \& M. F. Prevost. 1984. Zoochorie et premiers stades de la régénération naturelle après coupe en forêt guyanaise. Rev. Ecol. (Terre Vie) 39: 369-400.

Frankie, G. W. 1976. Pollination of widely dispersed trees by animals in Central America, with emphasis on bee pollination systems. Pp. 151-159 in J. Burley \& B. T. Styles (editors), Tropical Trees. Variation, Breeding, and Conservation. Academic Press, London.

Gleason, H. A. 1929. Studies on the flora of northern South America-XII. Cyphostyleae, a new tribe in Melastomataceae. Bull. Torrey Bot. Club 56: 97 112.

1932. A synopsis of the Melastomataceae of British Guiana. Brittonia 1: 127-184.

Goulding, M. 1980. The fishes and the forest. Univ. California Press, Berkeley, California.

Gustafsson, A. 1946-47. Apomixis in higher plants. Acta Univ. Lund. 42/43: 1-370.

Heithaus, E. R. 1979. Community structure of neotropical flower visiting bees and wasps: diversity and phenology. Ecology 60: 190-202.

HıCKEY, L. J. 1977. Stratigraphy and paleobotany of the Golden Valley Formation (early Tertiary) of western North Dakota. Geol. Soc. Amer. no. 150.

Hıtty, S. L. 1980. Flowering and fruiting periodicity in a premontane rain forest in Pacific Columbia. Biotropica 12: 292-306.

Huber, J. 1909 [1910]. Mattas e madeiras amazônicas. Bol. Mus. Paraense Hist. Nat. 6: 91-216.

JANZEN, D. H. 1971. Euglossine bees as long-distance pollinators of tropical plants. Science 171: 203-205.

Johnson, L. A. S. \& B. G. Briggs. 1984. Myrtales and Myrtaceae - a phylogenetic analysis. Ann. Missouri Bot. Gard. 71: 700-756.

Kaur, A., C. D. Ha, K. Jong, V. E. Sands, H. T. Chan, E. Soepadmo \& P. S. Ashton. 1978. Apomixis may be widespread among trees of the climax rain forest. Nature 271: 440-442.

Kral, R. \& P. E. Bostick. 1969. The genus Rhexia (Melastomataceae). Sida 3: 387-440.

Kubitzkı, K. 1978. Caraipa and Mahurea (Bonnetiaceae). Mem. New York Bot. Gard. 29: 82-138.

Kugler, H. 1970. Einfuehrung in die Bluetenoekologie. Fischer Vlg., Stuttgart.

Lagerheim, G. 1899. Ueber die Bestaeubungs- und Aussaeunungseinrichtungen von Brachytoum ledifolium (Desr.) Cogn. Bot. Not. 1899: 105-122.

LAROCA, S. 1970. Contribuição para o conhecimento das relaçǒes entre abelhas e flores: coleta de pólen das anteras tubulares de certas Melastomataceae. Revista Floresta 2: 69-74.

LEGGeT, W. H. 1881. Fertilization of Rhexia virginica L. Bull. Torrey Bot. Club 8: 102-104.
Linsley, E. G. \& M. A. Cazier. 1963. Further observations on bees which take pollen from plants of the genus Solanum. Pan-Pacific Entomol. 39: 1-18.

-, C. M. Rick \& S. G. STEPhEns. 1966. Observations on the floral relationships of the Galápagos carpenter bee. Pan-Pacific Entomol. 42: 1-18.

Lowry, J. B. 1976. Anthocyanins of the Melastomataceae, Myrtaceae and some allied families. Phytochemistry 15: 513-516.

Lumer, C. 1980. Rodent pollination of Blakea (Melastomataceae) in a Costa Rican cloud forest. Brittonia 32: $512-517$.

—. 1982. The pollination ecology, breeding sys. tems and phenology of Blakea and Topobea (Melastomataceae) in Monteverde, Costa Rica. Thesis, City University, New York, New York.

— \& R. D. SCHOER. 1986. Pollination of Blakea austin-smithii and B. penduliflora (Melastomataceae) by small rodents in Costa Rica. Biotropica 18: 363-364.

McMullen, C. K. 1985. Observations on insect visitors to flowering plants of Isla Santa Cruz. Part I. The endemic Carpenter bee. Not. Galapagos 42: 24-25.

Macior, L. W. 1970. Pollination ecology of Dodecatheon amethystinum (Primulaceae). Bull. Torrey Bot. Club 97: 150-153.

— 1971. Co-evolution of plants and animals systematic insights from plant-insect interactions. Taxon 20: 17-28.

Magnusson, W. E. \& T. M. Sanaiotti. 1987. Dispersal of Miconia seeds by the rat Bolomys lasiurus. J. Trop. Ecol. 3: 277-278.

Michener, C. D. 1962. An interesting method of pollen collecting by bees from flowers with tubular anthers. Revista Biol. Trop. 10: 167-175.

- 1979. Biogeography of the bees. Ann. Missouri Bot. Gard. 66: 277-347.

- M. L. Winston \& R. Jander. 1978. Pollen manipulation and related activities and structures in bees of the family Apidae. Univ. Kansas Sci. Bull. 51: 575-60l.

Mori, S. A. \& J. J. Pipoly. 1984. Observations on the big bang flowering of Miconia minutiflora (Mela. stomataceae). Brittonia 36: 337-34l.

Morley, T. 1953. The genus Mouriri (Melastomataceae). Univ. California Publ. Bot. 26: 223-312.

. 1963. Votomitia Aublet (Melastomataceae). Bull. Torrey Bot. Club 90: 1-16.

- 1976. Memecyleae (Melastomataceae). Fl. Neotrop. 15: 1-295.

1985. Five new taxa of New World Memecy. leae (Melastomataceae). Ann. Missouri Bot. Gard. 72: $548-557$.

Moure, J. S. 1970. The species of euglossine bees of Central America belonging to the subgenus Eugloss. ella (Hymenoptera, Apidae). Anais Acad. Brasil. Ci. 42: $147-157$

Mueller, F. 1873. (letters). In: A. Moeller (editor), Fritz Mueller. Werke, Briefe und Leben. Volumes I-III. Fischer Vlg., Jena.

- 1883. Two kinds of stamens with different functions in the same flower. Nature 27: 364-365.

Mueller, H. 1881. Two kinds of stamens with different functions in the same flower. Nature 24: 307-308.

- 1883. Arbeitstheilung bei Staubgefaessen von Pollenblumen. (Von Hermann Mueller mit Beobachtungen von Fritz Mueller.) Kosmos 13: 241-259.

NefF, J. L. \& B. B. Simpson. 1981. Oil-collecting 
structures in the Anthophoridae (Hymenoptera): morphology, function, and use in systematics. J. Kansas Entomol. Soc. 54: 95-123.

NetTancourt, D. DE. 1977. Incompatibility in Angiosperms. Springer, Berlin.

Opler, P. A., H. G. Baker \& G. W. Frankie. 1980. Plant reproductive characteristics during secondary succession in neotropical lowland forest ecosystems. Biotropica 12 (suppl.): 40-46.

Patel, V. C., J. J. Skvarla \& P. H. Raven. 1984. Pollen characters in relation to the delimination of Myrtales. Ann. Missouri Bot. Gard. 71: 858-969.

Percival, M. S. 1965. Floral Biology. Pergamon Press, Oxford.

Proctor, M. \& P. Yeo. 1972. The Pollination of Flowers. Taplinger Publ. Comp., New York.

Renner, S. S. 1983. The widespread occurrence of anther destruction by Trigona bees in Melastomataceae. Biotropica 15: 257-267.

- 1984. Phaenologie, Bluetenbiologie und Rekombinationssyteme einiger zentralamazonischer Melastomataceen. Doctoral Dissertation (printed copy), Univ. Hamburg, 1984.

. 1986. The neotropical epiphytic Melastomataceae: phytogeographic patterns, fruit types, and floral biology. Selbyana 9: 104-111.

. 1986/1987. Reproductive biology of Bellu. cia (Melastomataceae). Acta Amazonica 16/17: $197-$ 208.

2. 1987a. Seed dispersal. Progr. Botany 49: 413-432.

. 1987b. Sandemania hoehnei (Tibouchineae: Melastomataceae): taxonomy, distribution, and biology. Brittonia 39: 44l-446.

- Reproduction and evolution in some genera of neotropical Melastomataceae. In: G. Gottsberger \& G. T. Prance (editors), The Biology of Tropical Woody Angiosperms. New York Bot. Gard. (in press.)

- Floral biological observations on Heliamphora tatei (Sarraceniaceae) and other plants from Cerro de la Neblina in Venezuela. Pl. Syst. Evol. (In press.)

RICKLEFS, R. E. 1977. A discriminant function analysis of assemblages of fruit-eating birds in Central America. Condor 79: 228-231.

Roosmalen, M. G. M. van. 1985. Fruits of the Guianan Flora. Inst. Syst. Bot., Utrecht Univ., Netherlands.

RouвIK, D. W. 1979a. Competition studies of colonizing Africanized honeybees and native pollinators in South America. Thesis, Univ. Kansas, Lawrence, Kansas.

—. 1979b. Africanized honey bees, stingless bees, and the structure of tropical plant-pollinator communities. Proc. 4th International Symposium on Pollination, Maryland Agric. Exp. Sta. Misc. Publ. 1: 403-417.

— \& C. D. Michener. 1985. Nesting biology of Crawfordapis (Hymenoptera, Colletidae). J. Kansas Entomol. Soc. 57: 662-671.

Sakagami, S. F., M. J. Montenegro \& W. E. Kerr. 1965. Behavior studies of the stingless bees, with special reference to the oviposition process. J. Fac. Sci. Hokkaido Imp. Univ., Ser. 4, Zool. 15: 578607.

Skutch, A. F. 1980. Arils as food for tropical American birds. Condor 82: 31-42.

SNow, D. W. 1965. A possible selective factor in the evolution of fruiting seasons in tropical forest. Oikos 15: $274-281$.
1971. Evolutionary aspects of fruit-eating by birds. Ibis 113: 194-202.

1981. Tropical frugivorous birds and their food plants: a world survey. Biotropica 13: 1-14.

- \& B. K. SNOW. 1980. Relationships between hummingbirds and flowers in the Andes of Colombia. Bull. Brit. Mus. (Nat. Hist.) Zool. 38: 105-139.

Sobrevila, C. \& M. T. K. Arroyo. 1982. Breeding systems in a montane cloud forest in Venezuela. Pl. Syst. Evol. 140: 19-37.

Solt, M. L. \& J. J. Wurdack. 1980. Chromosome numbers in the Melastomataceae. Phytologia 47: 199-220.

Sourd, C. \& A. Gautier-Hion. 1986. Fruit selection by a forest guenon. J. Anim. Ecol. 55: 235-244.

Subramanyam, K. 1942. Gametogenesis and embry. ogeny in a few members of the Melastomataceae. J. Indian Bot. Soc. 21: 68-85.

1944. A contribution to the life-history of Sonerila wallachii Benn. Proc. Indian Acad. Sci. 19: 115-120.

- 1948. An embryological study of Melastoma malabathricum L. J. Indian Bot. Soc. 27: 11-19.

Symon, D. E. 1979. Sex forms in Solanum (Solanaceae) and the role of pollen collecting insects. Pp. 385397 in J. G. Hawkes, R. N. Lester \& A. D. Skelding (editors), The Biology and Taxonomy of the Solanaceae. Academic Press, London.

ThorP, R. W. 1979. Structural, behavioral, and physiological adaptations of bees (Apoidea) for collecting pollen. Ann. Missouri Bot. Gard. 66: 788-812.

\& J. R. EsTES. 1975. Intrafloral behavior of bees on flowers of Cassia fasciculata. J. Kansas Entomol. Soc. 48: 175-184.

Tobe, H. \& P. H. Raven. 1984. The number of cells in the pollen of Melastomataceae (Myrtales). Bot. Mag. (Tokyo) 97: 131-136.

Triana, J. 1867. Melastomaceae. Pp. 725-773 in G. Bentham \& J. D. Hooker, Genera Plantarum. Reeve \& Co., London.

Troll, W. 1922. Ueber Staubblatt- und Griffelbewegungen und ihre teleologische Deutung. Flora 15: 19l-250, pl. IV-VI.

UlE, E. 1895. Ueber die Bluetheneinrichtungen von Purpurella cleistoflora, einer neuen Melastomatcee. Ber. Deutsch. Bot. Ges. 13: 415-420.

1896. Weiteres zur Bluetheneinrichtung von Purpurella cleistopetala und Verwandten. Ber. Deutsch. Bot. Ges. 14: 169-178.

. 1909. Itatiaia. Bot. Jahrb. Syst. 42: 234235.

VAN DER PIJL, L. 1939. Over de meeldraden van enkele Melastomataceae. Trop. Natuur 28: 169-172.

- 1954. Xylocopa and flowers in the tropics. I-III. Proc. Kon. Ned. Akad. Wetensch., Ser. C 57: 413-423, 541-562.

Vliet, G. J. C. M. van, J. Koek-Noorman \& B. J. H. TER WELLE. 1981. Wood anatomy, classification and phylogeny of the Melastomataceae. Blumea 27: 463-473.

VOGEL, S. 1957. Fledermausblumen in Suedamerika. Oesterr. Bot. Z. 104: 491-530.

- 1962 [1963]. Duftdruesen im Dienste der Bestaeubung. Ueber Bau und Funktion der Osmophoren. Akad. Wiss. Abh. Math.-Naturviss. Kl. 10: 601-763.

—. 1966. Parfuemsammelnde Bienen als Be- 
staeuber von Orchidaceen und Gloxinia. Oesterr. Bot. Z. 113: 302-361.

1974. Oelblumen und oelsammelnde Bienen Pp. 284-547 in W. Rauh (editor), Tropische und subtropische Pflanzenwelt. 7. Steiner, Wiesbaden.

- 1978. Evolutionary shifts from reward to deception in pollen flowers. Pp. 89-96 in A. J. Richards (editor), The Pollination of Flowers by Insects. Academic Press, London, New York.

- 1988. Neu er kannte bzw. new dokumentierte Fledermausblumen aus drei Kontinentem. Tagungsberichte Deutsche Bot. Ges.) Giessen 1988: 188.

WaRNER, R. 1981. Systematics of Central American Monolena (Melastomataceae). Thesis, Univ. Minnesota, St. Paul, Minnesota.

Welle, B. J. H. Ter \& J. Koek-Noorman. 1981. Wood anatomy of the neotropical Melastomataceae. Blumea 27: 335-394.

Wheelwright, N. T., W. A. Haber, K. G. Murray \& C. Guindon. 1984. Tropical fruit-eating birds and their food plants: a survey of a Costa Rican lower montane forest. Biotropica 16: 173-192.

Whiffin, T. 1973. Analysis of a hybrid swarm between Heterocentron elegans and H. glandulosum (Melastomataceae). Taxon 22: 413-423.

— \& A. S. Томв. 1972. The systematic signifi- cance of seed morphology in the neotropical capsularfruited Melastomataceae. Amer. J. Bot. 59: 41 1422.

Wurdack, J. J. 1954. A revision of the genus Brach. yotum (Tibouchineae - Melastomataceae). Mem. New York Bot. Gard. 8: 343-407.

1964. Melastomataceae. Pp. 135-186 in B. Maguire, J. J. Wurdack, and collaborators, The Botany of the Guayana Highland-Part V. Mem. New York Bot. Gard. 10.

- 1965. Certamen Melastomataceis. XI. Phytologia 11: 377-400.

- 1980. Melastomataceae. In G. Harling \& B. Sparre (editors), Flora of Ecuador. 13: 1-406. Swed ish Natural Science Research Council, Stockholm.

- 1986. Atlas of hairs for neotropical Melas. tomataceae. Smithsonian Contr. Bot. 63: 1-80.

\& R. KRAl. 1982. The genera of Melastomataceae in the Southeastern United States. J. Arnold Arbor. 63: 429-439.

Ziegler, A. 1925. Beitraege zur Kenntnis des Androeceums und der Samenentwicklung einiger Melastomataceen. Bot. Arch. 9: 398-467.

Zimmermann, J. G. 1932. Ueber die extrafloralen Nektarien der Angiospermen. Beih. Bot. Centralbl. 49: 99-196. 


\section{$2 \mathrm{BHL}$ Biodiversity Heritage Library}

Renner, Susanne. 1989. "A Survey of Reproductive Biology in Neotropical Melastomataceae and Memecylaceae." Annals of the Missouri Botanical Garden 76, 496-518. https://doi.org/10.2307/2399497.

View This Item Online: https://www.biodiversitylibrary.org/item/89020

DOI: https://doi.org/10.2307/2399497

Permalink: https://www.biodiversitylibrary.org/partpdf/5355

\section{Holding Institution}

Missouri Botanical Garden, Peter H. Raven Library

\section{Sponsored by}

Missouri Botanical Garden

\section{Copyright \& Reuse}

Copyright Status: In copyright. Digitized with the permission of the rights holder.

License: http://creativecommons.org/licenses/by-nc-sa/3.0/

Rights: https://biodiversitylibrary.org/permissions

This document was created from content at the Biodiversity Heritage Library, the world's largest open access digital library for biodiversity literature and archives. Visit BHL at https://www.biodiversitylibrary.org. 